\title{
Dynamics of a Turbulent Buoyant Plume in a Stratified Fluid: An Idealized Model of Subglacial Discharge in Greenland Fjords $\mathscr{O}$
}

\author{
EKATERINA EZHOVA ${ }^{a}$ \\ Linné FLOW Centre, and Swedish e-Science Research Centre, Department of Mechanics, Royal Institute of Technology, Stockholm, Sweden \\ Claudia Cenedese \\ Physical Oceanography Department, Woods Hole Oceanographic Institution, Woods Hole, Massachusetts \\ LUCA BRANDT \\ Linné FLOW Centre, and Swedish e-Science Research Centre, Department of Mechanics, Royal Institute of Technology, Stockholm, Sweden
}

(Manuscript received 30 November 2016, in final form 6 July 2017)

\begin{abstract}
This study reports the results of large-eddy simulations of an axisymmetric turbulent buoyant plume in a stratified fluid. The configuration used is an idealized model of the plume generated by a subglacial discharge at the base of a tidewater glacier with an ambient stratification typical of Greenland fjords. The plume is discharged from a round source of various diameters and characteristic stratifications for summer and winter are considered. The classical theory for the integral parameters of a turbulent plume in a homogeneous fluid gives accurate predictions in the weakly stratified lower layer up to the pycnocline, and the plume dynamics are not sensitive to changes in the source diameter. In winter, when the stratification is similar to an idealized two-layer case, turbulent entrainment and generation of internal waves by the plume top are in agreement with the theoretical and numerical results obtained for turbulent jets in a two-layer stratification. In summer, instead, the stratification is more complex and turbulent entrainment by the plume top is significantly reduced. The subsurface layer in summer is characterized by a strong density gradient and the oscillating plume generates internal waves that might serve as an indicator of submerged plumes not penetrating to the surface.
\end{abstract}

\section{Introduction}

According to recent observations (Gardner et al. 2013), about one-third of sea level rise is due to glacier melting. The evidence is that melting increased significantly during the past two decades (Shepherd et al. 2012), leading to an anomalous freshwater input into the North Atlantic (Bamber et al. 2012) that is expected to influence the Atlantic meridional overturning circulation. Accordingly, mass loss from Antarctica and the Greenland Ice Sheets and the associated freshwater

\footnotetext{
${ }^{\text {a }}$ Current affiliation: Department of Physics, University of Helsinki, Helsinki, Finland.

Supplemental information related to this paper is available at the Journals Online website: https://doi.org/10.1175/ JPO-D-16-0259.s1.
}

Corresponding author: Ekaterina Ezhova, ekaterina.ezhova@ helsinki.fi fluxes have to be accounted for in numerical models predicting climate variability on the time scale of dozens of years (Straneo and Cenedese 2015).

Mass loss from the Greenland Ice Sheet can be partly attributed to melting and calving of large, marineterminating outlet glaciers. Recent investigations have shown that two of the main factors controlling submarine melting at the glacier fronts are the ambient oceanic stratification and subglacial discharge-the meltwater from the glacier surface in contact with air percolating through the glacier and discharged at the glacier base (Sciascia et al. 2013; Xu et al. 2012, 2013). Subglacial discharge generates turbulent buoyant plumes propagating vertically next to the glacier front and enhancing submarine melting at the termini of a glacier (Cenedese and Gatto 2016a; McConnochie and Kerr 2017).

The importance of subglacial discharge for submarine melting has been recognized only recently and the 
investigations of the near-glacier dynamics are sparse. Almost all these recent studies report results of numerical modeling employing large-scale ocean-ice models (Sciascia et al. 2013; Kimura et al. 2014; Slater et al. 2015). Large-scale models parameterize both melting rates and turbulent entrainment and the results are very sensitive to these parameterizations (Straneo and Cenedese 2015). Therefore, understanding the physics of the near-glacier phenomena is vital for accurate predictions of melting.

The effects of subglacial discharge on glacier melting are difficult to explore in the field; the sources of buoyant fluid at the base of tidewater glaciers are largely uncertain. Experimental and numerical investigations of submarine melting influenced by subglacial discharge usually employ a source of buoyant fluid adjacent to the glacier ice face, and the results are often compared to plume theory (Slater et al. 2015; Cenedese and Gatto 2016a,b; McConnochie and Kerr 2017). The classical theoretical plume model by Morton et al. (1956) is based on the equations of conservation of mass, buoyancy, and momentum integrated over the cross section of the plume (referred to hereinafter as MTT theory). This theory has been developed for the plumes in a homogeneous fluid but has been used successfully also to estimate the penetration height of plumes in a uniformly stratified fluid (Slater et al. 2015). An MTTbased theoretical model of melting for a line source of subglacial discharge has been developed by Jenkins (2011). A similar model for a discrete channel of discharge is described by Cowton et al. (2015). These models, however, rely on several parameters that are poorly constrained in the presence of a vertical wall. The drag and entrainment coefficients might be affected by three-dimensional, near-wall turbulence, while entrainment can also be influenced by ambient stratification. Though some progress has been made in quantifying entrainment coefficients for plumes in a homogeneous medium (van Reeuwijk et al. 2016; Burridge et al. 2016), the influence of stratification is still not clear. The focus of this work is to consider the effects of stratifications typical of Greenland fjords on the dynamics of a buoyant plume generated by a realistic subglacial discharge exiting from a round source. Thus, our model is a vertical axisymmetric plume in a stratified fluid without the presence of a wall. The absence of a wall allows us to investigate the effects of complex stratification on the plume dynamics separately from the wall influence, to predict what dynamics can be expected, and further to see how these dynamics will be modified by the presence of a wall and, ultimately, a melting wall. The influence of the wall on the plume dynamics will be the focus of a future study.
A typical density stratification in Greenland fjords is weakly linear in winter with a narrow pycnocline at depth of $\sim 160 \mathrm{~m}$ and more complicated in summer (Fig. 1). Typically, a moderate discharge (defined quantitatively in section 2) produces a plume, which does not reach the free surface and intrudes at a depth. The well-mixed plume at the pycnocline entrance penetrates into the lighter fluid above because of the excess of momentum and forms a fountain with a Froude number of order one (weak fountain), for which the MTT theory does not hold (Kaye 2008; Ezhova et al. 2016). Moreover, both winter and summer stratifications (Fig. 1) are characterized by length scales comparable to the diameter of the plume close to the pycnocline. The thickness of the pycnocline is an important parameter, influencing the penetration height, turbulent entrainment by the plume top, and the shape of the plume top. These aspects define how large the "fountain part" is in comparison to the "plume part," how large the velocities in the fountain are, and, overall, whether it is important to account for the fountain formation in the estimates of melting rates. Here, we quantify the turbulent entrainment by the top of the plume for winter and summer stratifications and comment on the expected consequences for melting.

Furthermore, the vertical oscillations of a weak fountain in a stratified fluid are able to generate internal waves (Troitskaya et al. 2008; Ezhova et al. 2016), which might be important for two reasons. First, internal waves are capable to mix the ambient fluid; second, they can be manifested on the free surface, especially for shallow pycnoclines, and thus remote sensing may prove a useful tool to reveal the location of submerged plumes. Therefore, we discuss in what follows the plume nonstationary effects, including plume oscillations and generation of internal waves.

The present study employs large-eddy simulations (LES) focusing on the dynamics of a vertical turbulent plume in a fluid having a stratification identical to that observed in Sermilik Fjord near Helheim Glacier, a major Greenland outlet glacier. Temperature and salinity profiles in winter and summer have been measured by Straneo et al. (2011). This fjord has a total depth of $600 \mathrm{~m}$, in the middle of the range $300-800 \mathrm{~m}$ reported for tidewater glaciers (Straneo and Cenedese 2015). The stratification observed, with Atlantic salty and warm waters at the bottom and fresher and cold polar waters at the top, has been detected in several fjords in Greenland (Straneo et al. 2012).

The numerical setup is described in section 2, whereas the properties of the buoyant plumes generated by a subglacial discharge in a typical Greenland fjord for two 


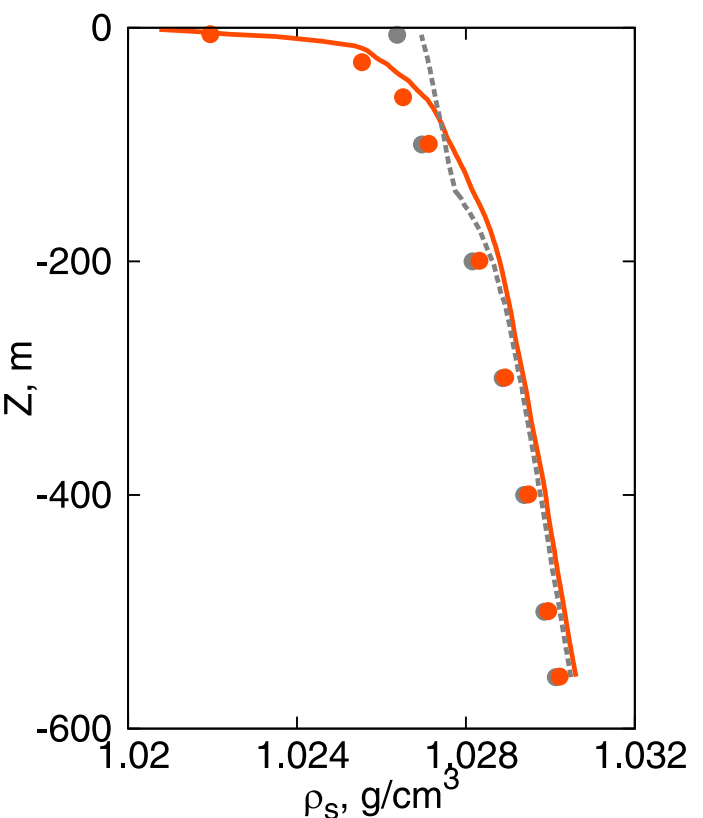

summer winter

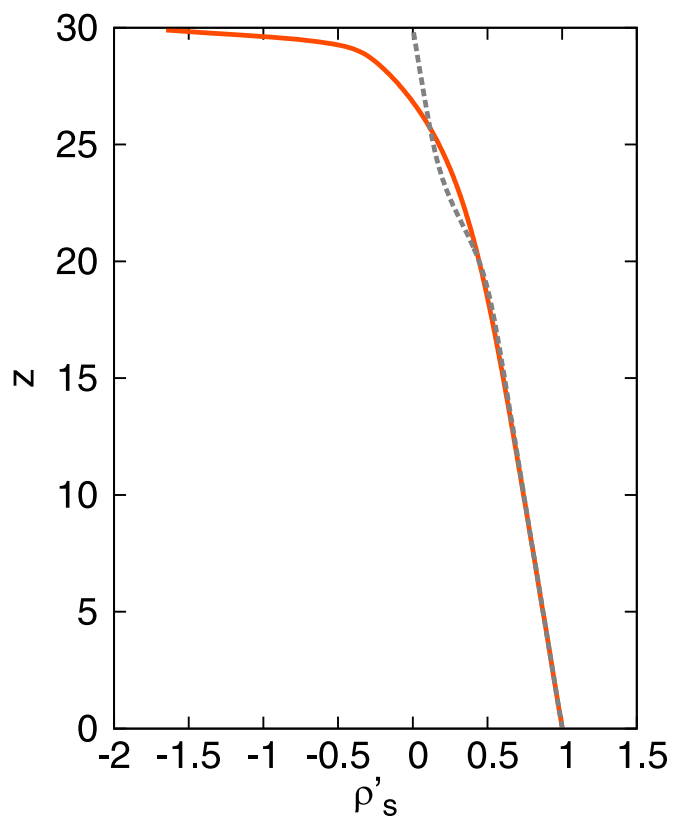

summer winter

FIG. 1. (left) Vertical density profiles based on the temperature and salinity profiles measured in Sermilik Fjord in summer and winter by Straneo et al. (2011). Curves correspond to the reduced seawater state equation [(1)]; symbols refer to the full seawater state equation. (right) Analytical functions used for the approximation of the density profiles in the simulations (see appendix B). Summer stratification (orange), winter stratification (gray).

different source diameters and the winter and summer stratifications are discussed in section 3 . We quantify mean penetration depth and turbulent entrainment and investigate the generation of internal waves by the plumes.

\section{Model description and numerical setup}

In the field, a plume generated by a subglacial discharge is expected to have some initial horizontal momentum; however, it reattaches to the glacier face close to the source location because of the Coanda effect (Kimura et al. 2014). Thus, we neglect the initial horizontal momentum of the plume and assume that its influence on the plume dynamics is small and it is important only close to the source (Kimura et al. 2014; Cenedese and Gatto 2016b). Moreover, idealized models have considered the plume adjacent to the glacier face as a half-conical plume (Cenedese and Gatto 2016b; Mankoff et al. 2016). Thus, to compare the results for a half-conical plume with MTT theory, developed for axisymmetric plumes and entraining ambient fluid from all directions, it is convenient to add the second halfconical part and consider an axisymmetric plume with double the total discharge as a model (Mankoff et al. 2016). We follow this approach and use an axisymmetric vertical turbulent plume in a complex stratification as an idealized model of the plume generated by a subglacial discharge. Given the estimate of the total subglacial discharge for Helheim Glacier in Sermilik Fjord, $Q_{\text {tot }}=174 \mathrm{~m}^{3} \mathrm{~s}^{-1}$ (Sciascia et al. 2013), we perform simulations of an axisymmetric turbulent buoyant plume with source volume flux of $Q_{0 i}=174 \times 2 \mathrm{~m}^{3} \mathrm{~s}^{-1}$ (referred to hereinafter as the basic discharge), in order to investigate its dynamics in typical winter and summer stratifications in Greenland fjords (Straneo et al. 2011). The subscript $i=1,2$ corresponds to the specific simulation setup (see Table 1). This basic discharge, in the presence of an ice face, produces a "convection-driven melting" regime (Jenkins 2011), where the contribution of submarine melting to the plume buoyancy is small. However, for a small discharge $\sim 10 \mathrm{~m}^{3} \mathrm{~s}^{-1}$ (Mankoff et al. 2016), the effect of submarine melting on the plume buoyancy flux cannot be neglected, and therefore we do not consider plumes generated by a small discharge in the present study. Furthermore, we do not consider a large discharge either ( $\gtrsim 400 \mathrm{~m}^{3} \mathrm{~s}^{-1}$ in the stratification considered here), which generates a plume penetrating to the free surface, and in these circumstances the stratification is not expected to play a significant role for the plume's dynamics. We refer to a discharge as moderate if it is neither small nor large.

Based on the estimates of a source channel size from field experiments, summarized by Kimura et al. (2014), 
TABLE 1. Parameters used in the different simulations.

\begin{tabular}{cccccc}
\hline \hline Case & Diameter $(\mathrm{m})$ & Discharge $\left(\mathrm{m}^{3} \mathrm{~s}^{-1}\right)$ & Initial velocity $\left(\mathrm{m} \mathrm{s}^{-1}\right)$ & Froude number & Stratification \\
\hline 1 & $D_{01}=20$ & $Q_{01}=174 \times 2$ & $W_{01}=1.1$ & $\mathrm{Fr}_{01}=0.47$ & Summer \\
2 & $D_{01}=20$ & $Q_{01}=174 \times 2$ & $W_{01}=1.1$ & $\mathrm{Fr}_{01}=0.47$ & Winter \\
3 & $D_{02}=10$ & $Q_{02}=174 \times 2$ & $W_{02}=4.4$ & $\mathrm{Fr}_{02}=2.70$ & Winter \\
4 & $D_{03}=10$ & $Q_{03}=174 \times 3$ & $W_{03}=6.2$ & $\operatorname{Fr}_{03}=3.80$ & Summer \\
5 & $D_{03}=10$ & $Q_{03}=174 \times 3$ & $W_{03}=6.2$ & $\operatorname{Fr}_{03}=3.80$ & Winter \\
\hline
\end{tabular}

we consider for the basic discharge a round source of diameter $D_{01}=20 \mathrm{~m}$ for winter and summer stratification and $D_{02}=10 \mathrm{~m}$ for winter stratification. We have also performed two additional simulations with source diameter $D_{03}=10 \mathrm{~m}$ and an increased discharge $Q_{03}=174 \times 3 \mathrm{~m}^{3} \mathrm{~s}^{-1}$, both in winter and summer. We choose to increase the basic discharge because the difference in stratification between summer and winter becomes significant near the free surface, and hence it is expected to have a noticeable impact on the plume dynamics, as the plume penetrates closer to the free surface for increased discharges. Table 1 summarizes the parameters of the five simulations. The initial velocities are estimated by $W_{0 i}=4 Q_{0 i} / \pi D_{0 i}^{2}(i=1,2,3$; see Table 1). The Froude number at the inflow is $\mathrm{Fr}_{0 i}=W_{0 i} / \sqrt{g_{0}^{\prime} D_{0 i}}$, where $g_{0}^{\prime}$ is the reduced gravity based on the density difference between the source water and the ambient fluid at the bottom.

Following Hunt and Kaye (2001, 2005), we introduce the source parameter $\Gamma_{0 i} \propto 1 / \mathrm{Fr}_{0 i}^{2}$ and classify the plume caused by the basic discharge from the source of $20-\mathrm{m}$ diameter, $\Gamma_{01} \approx 4.4$, as a lazy plume and the plume from the source of $10-\mathrm{m}$ diameter, $\Gamma_{02} \approx 0.13$, as a forced plume. Thus, depending on the initial source diameter, we obtain either a buoyancy-driven lazy plume or a momentum-driven forced plume. The simulations with increased discharge are characterized by $\Gamma_{03} \approx 0.07$, resulting in forced plumes.

Stratifications representative of Greenland fjords in winter and summer are chosen as follows: Based on the salinity and temperature distributions measured in the Sermilik Fjord in summer and winter, as reported by Straneo et al. (2011), we obtain the corresponding density profiles using the following simplified state equation for the seawater (Gill 1982):

$$
\rho_{s}=0.99984+0.001\left(\varepsilon_{1} S+\varepsilon_{2} T-\varepsilon_{3} S T-\varepsilon_{4} T^{2}+\varepsilon_{5} Z\right),
$$

where $\rho_{s}$ is density $\left(\mathrm{g} \mathrm{cm}^{-3}\right), S$ is salinity $\left(\mathrm{g} \mathrm{kg}^{-1}\right), T$ is temperature $\left({ }^{\circ} \mathrm{C}\right), Z$ is depth $(\mathrm{m})$, and the coefficients $\varepsilon_{1}=0.8245, \varepsilon_{1}=0.0679, \varepsilon_{1}=0.00409, \varepsilon_{1}=0.00909$, and $\varepsilon_{5}=0.00450$.
The vertical density profiles calculated using (1) are depicted in Fig. 1 (left panel). The analytical functions used for the approximation of these density profiles can be found in appendix B and are shown in Fig. 1 (right panel). The average depth of the fjord near the glacier is $600 \mathrm{~m}$. The winter profile is close to linear with a weakly pronounced pycnocline at $160-\mathrm{m}$ depth. The profile typical of summer is characterized by a wide (up to $160 \mathrm{~m}$ ), well-pronounced pycnocline with a sharper density gradient in the proximity of the surface.

The dynamics of a plume in a stratified fluid are governed by the Navier-Stokes equations for an incompressible fluid with the Boussinesq approximation to model the buoyancy effects and a conservation equation for the density field. Given the spatial scales considered here, we resort to LES to reduce the computational costs. In a LES, the large turbulent eddies are fully resolved, whereas the effect of the smallest scales, those not resolved on the computational mesh, is modeled. We performed simulations employing the dynamic Smagorinsky model (Smagorinsky 1963; Germano et al. 1991) in the spectral element code Nek5000 (Fischer et al. 2008). The LES model in the code Nek5000 used in this study has demonstrated its reliability in predicting the dynamics of jet flows in stably stratified fluids in our previous study (Ezhova et al. 2016) and has been successfully applied to the modeling of stratified fluids in several works (see Özgökmen and Fischer 2008; Özgökmen et al. 2009; Fabregat et al. 2016; and references therein). The additional validation of the LES model for the case of a lazy plume in a homogeneous fluid is given in appendix A.

In LES, a filter is applied to derive a system of equations for the resolved scales that reads in dimensionless form and in a Cartesian coordinate system

$$
\begin{aligned}
\frac{\partial u_{x}}{\partial t}+(\mathbf{u}, \nabla) u_{x}= & -\frac{\partial p}{\partial x}+\frac{1}{\operatorname{Re}_{i}} \Delta u_{x} \\
& -\frac{1}{\operatorname{Re}_{i}}\left(\frac{\partial \tau_{x x}}{\partial x}+\frac{\partial \tau_{x y}}{\partial y}+\frac{\partial \tau_{x z}}{\partial z}\right),
\end{aligned}
$$




$$
\begin{aligned}
\frac{\partial u_{y}}{\partial t}+(\mathbf{u}, \nabla) u_{y}= & -\frac{\partial p}{\partial y}+\frac{1}{\operatorname{Re}_{i}} \Delta u_{y} \\
& -\frac{1}{\operatorname{Re}_{i}}\left(\frac{\partial \tau_{y x}}{\partial x}+\frac{\partial \tau_{y y}}{\partial y}+\frac{\partial \tau_{y z}}{\partial z}\right), \\
\frac{\partial u_{z}}{\partial t}+(\mathbf{u}, \nabla) u_{z}= & -\frac{\partial p}{\partial z}+\frac{1}{\operatorname{Re}_{i}} \Delta u_{z}-\frac{1}{\operatorname{Fr}_{i}^{2}}\left(\rho-\rho_{s}^{\prime}\right) \\
& -\frac{1}{\operatorname{Re}_{i}}\left(\frac{\partial \tau_{z x}}{\partial x}+\frac{\partial \tau_{z y}}{\partial y}+\frac{\partial \tau_{z z}}{\partial z}\right), \\
\frac{\partial \rho}{\partial t}+(\mathbf{u}, \nabla) \rho= & \frac{1}{\operatorname{Re}_{i} \operatorname{Pr}} \Delta \rho \\
& -\frac{1}{\operatorname{Re}_{i}}\left(\frac{\partial \Theta_{x}}{\partial x}+\frac{\partial \Theta_{y}}{\partial y}+\frac{\partial \Theta_{z}}{\partial z}\right),
\end{aligned}
$$

and

$$
\frac{\partial u_{x}}{\partial x}+\frac{\partial u_{y}}{\partial y}+\frac{\partial u_{z}}{\partial z}=0
$$

The equations are made dimensionless with the largest source diameter $D_{0}=D_{01}=20 \mathrm{~m}$, the plume inflow velocity $W_{0 i}$, and the density difference between the surface and bottom waters in winter $\Delta \rho$ (see appendix B for more detail). We define the profile of stratification as $\rho_{s}^{\prime}=\left(\rho_{s}-\rho_{\text {ref }}\right) / \Delta \rho$, where $\rho_{s}$ is the undisturbed density profile and $\rho_{\text {ref }}$ is the reference density (the density on the sea surface in winter). The Reynolds number used in (2)-(6) is $\operatorname{Re}_{i}=\left(W_{0 i} D_{0}\right) / \nu$; the Froude number $\mathrm{Fr}_{i}=W_{0 i} / \sqrt{g^{\prime} D_{0}}$, with $g^{\prime}=(g \Delta \rho) / \rho_{\text {ref }}$ as the reduced gravity; and the Prandtl number $\operatorname{Pr}=\nu / \kappa$, where $\nu$ is the fluid kinematic viscosity and $\kappa$ is the coefficient of scalar diffusivity. Note that Froude numbers $\mathrm{Fr}_{i}$ are different from the source Froude numbers $\mathrm{Fr}_{0 i}$, and they are introduced for the convenience of simulations.

The fluxes representing the subgrid Reynolds stresses and the turbulent scalar transport are $\tau_{i j}=-\nu_{t}\left[\left(\partial u_{i} / \partial x_{j}\right)+\right.$ $\left.\left(\partial u_{j} / \partial x_{i}\right)\right]$ and $\Theta_{j}=-\left(\nu_{t} / \operatorname{Pr}_{t}\right)\left(\partial \rho / \partial x_{j}\right)$, respectively (using the standard index notations). The Reynolds number of the geophysical plumes modeled here is on the order of millions; however, the LES have $\mathrm{Re}=\mathrm{Re}_{i}=15000$ and assume that the flow at this Re captures the main features of the geophysical turbulent plume. This value is above the critical Re that must be exceeded to sustain three-dimensional inertially dominated turbulent fluctuations in a shear flow, which mark the so-called mixing transition (Dimotakis 2005). The turbulent Prandtl number is $\operatorname{Pr}_{t}=1$.

The schematic of the simulation is shown in Fig. 2. The simulations are performed in a rectangular domain of size $94 \times 94 \times 30$ in the horizontal and vertical directions $(1900 \mathrm{~m} \times 1900 \mathrm{~m} \times 600 \mathrm{~m}$ in dimensional units $)$

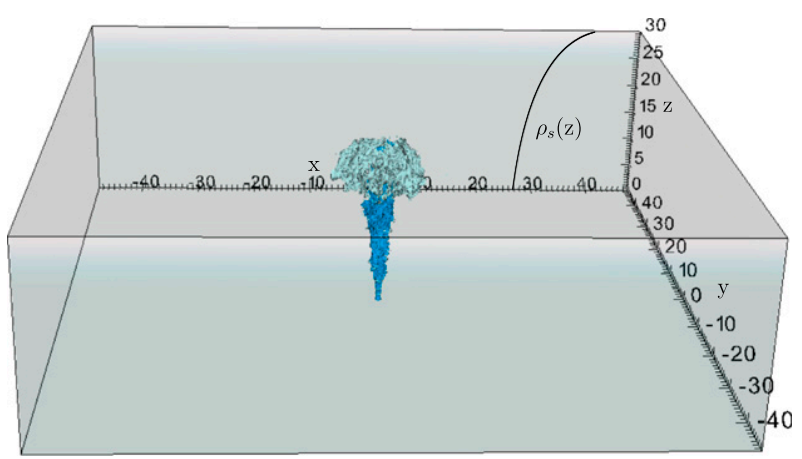

FIG. 2. Schematic of the simulations.

with a total number of spectral elements $48 \times 48 \times 48$. The polynomial order used in the simulations is 7 . We use a stretched grid with a high resolution at the inflow and in the regions of high density gradients. The size of the smallest element at the inflow is $0.15 \times 0.15 \times 0.4$, corresponding to a horizontal distance between the grid points of $\sim 0.02$ ( $40 \mathrm{~cm}$ in dimensional variables). The highest resolution in the vertical direction, used in the vicinity of the source and in the pycnocline, is estimated to be $\approx 60 \mathrm{~cm}$. The time step varied for the different simulations between $10^{-3}$ and $3 \times 10^{-3}$ time units to keep the CFL number around 0.4. Each simulation was performed on 512 cores. The average time for one simulation was around 7-8 days and the approximate total number of time steps was 750000 .

The boundary conditions at the inflow are top-hat velocity and density profiles with a smooth transition at the edge of the source, located at $r=\sqrt{x^{2}+y^{2}}=0.5$ for the source diameter $D_{01}=20 \mathrm{~m}\left(r=0.25\right.$ for $D_{02}=$ $D_{03}=10 \mathrm{~m}$ ) in the form of a hyperbolic tangent with a width $d=0.05$ in order to avoid numerical errors caused by the discontinuities in the shear layer. At the lateral boundaries we prescribe open boundary conditions for the horizontal velocity with a sponge layer of length seven eliminating the density fluctuations and vertical velocity. On the surface we use a "rigid-lid" approximation, allowing for nonzero horizontal velocity and setting the vertical velocity to zero.

\section{Results}

\section{a. Mean velocity and density}

In all simulations, the plume rises up to the pycnocline and penetrates through the level of neutral buoyancy caused by the excess of momentum. It then bounces back because of the negative buoyancy and propagates horizontally as a gravity current at the level of neutral buoyancy. To obtain the mean fields from the 

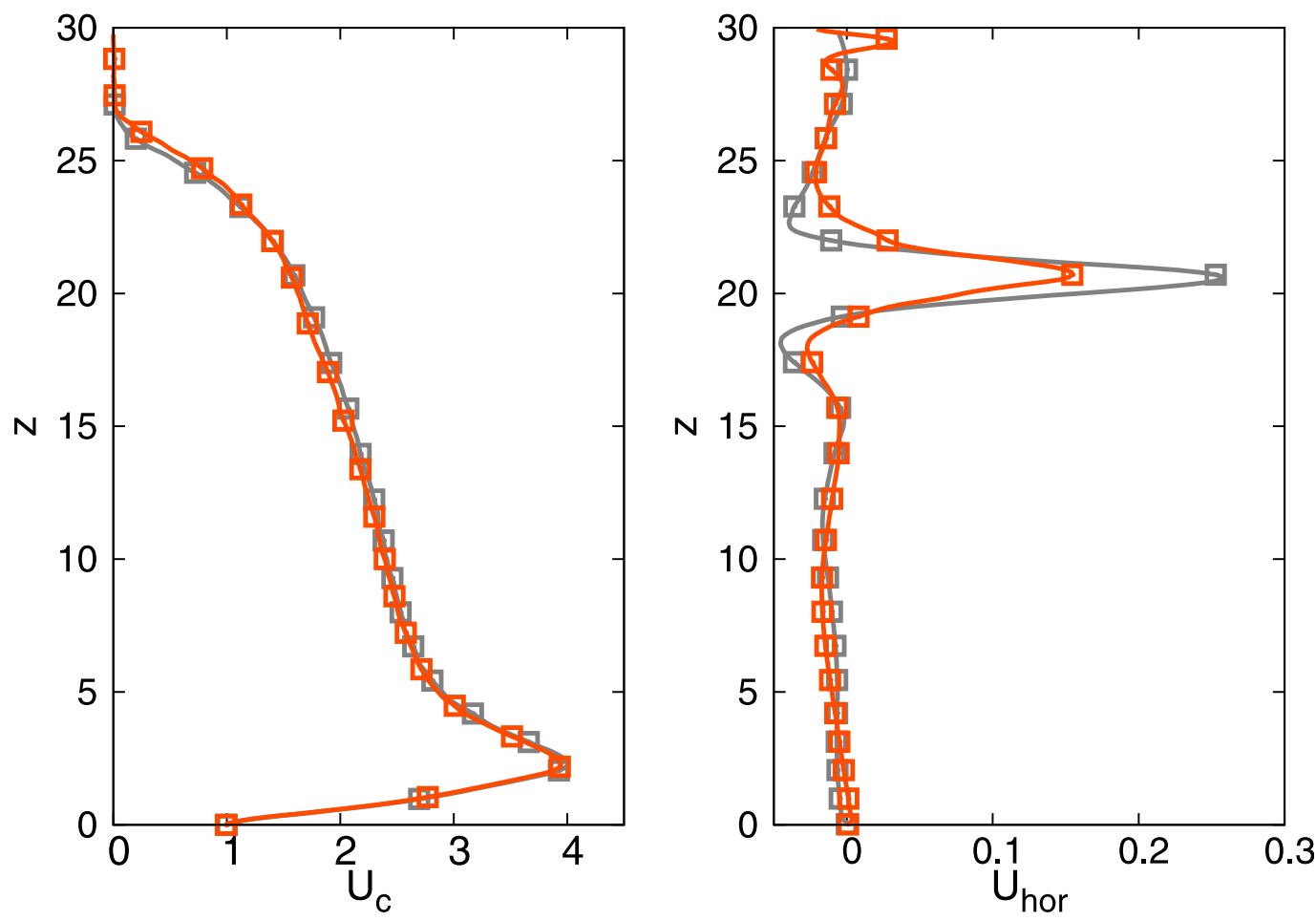

FIG. 3. (left) Mean vertical velocity along the plume axis. (right) Mean horizontal velocity at $r=20$. Lazy plume: summer stratification (orange), winter stratification (gray).

simulations, we run each case until the horizontal flow reaches the lateral boundaries and then start to gather statistics. We average the flow over not less than 10 periods of the propagating internal waves in the pycnocline, corresponding to approximately 350 nondimensional time units for cases 1 and 2, 1200 units for case 3 , and 1500 units for cases 4 and 5 (Table 1). Since the governing equations are nondimensionalized using the time scales pertaining to the plume (initial velocity $W_{0 i}$, which is different for different cases and the same spatial scale $D_{0}=20 \mathrm{~m}$ ), while averaging is performed over the time intervals related to internal waves, the nondimensional time intervals differ for different simulations.

The left panel of Fig. 3 shows the nondimensional mean vertical velocity along the plume axis $U_{c}$ for the lazy plume in summer and winter stratifications (cases 1 and 2 in Table 1). Both plumes penetrate the same distance to $h_{m} \approx 27$. The isocontours in the upper panel of Fig. 4 display the magnitude of the average velocity of the lazy plume for both stratifications, with the two panels looking very similar, which is not surprising given that the stratifications differ significantly only close to the free surface. However, there is a small but noticeable difference in the horizontal flow around $z=20$, which is slower and thicker in summer (see the vertical profiles of the nondimensional radial horizontal velocity, $U_{\text {hor }}$ in the right panel of the Fig. 3). Two mechanisms can be responsible for this: the difference in turbulent entrainment by the top of the plume in summer and winter and the difference in the two stratifications near $z=22-23$.

Figure 5 (left) shows the mean vertical velocities $U_{c}$ and densities $\rho_{c}$ along the plume axis in winter for the lazy and forced plumes (cases 2 and 3 in Table 1). We rescale the velocity for the lazy plume so that the nondimensional velocity $U=1$ in this figure corresponds to the inflow velocity for a forced plume of $4.4 \mathrm{~m} \mathrm{~s}^{-1}$ in dimensional units. The velocity and density profiles follow each other closely starting from $z \approx 7$, so the velocity and density scales at the entrance to the pycnocline are the same. Together with the similarity of the plume diameters (Fig. 5, right), this gives similar dynamics of the plumes in the pycnocline as well as similar stratified entrainment and generation of internal waves of the same frequency (as will be discussed later). Given that lazy and forced plumes in the same stratification have similar behavior after $z=7$, we choose to not simulate a forced plume with the summer stratification since we expect the plume dynamics to be the same as that of a lazy plume in summer (case 1 in Table 1).

Figure 5 (right) displays the dependence of the plume radius $b_{w}$ on the vertical coordinate in winter stratification 

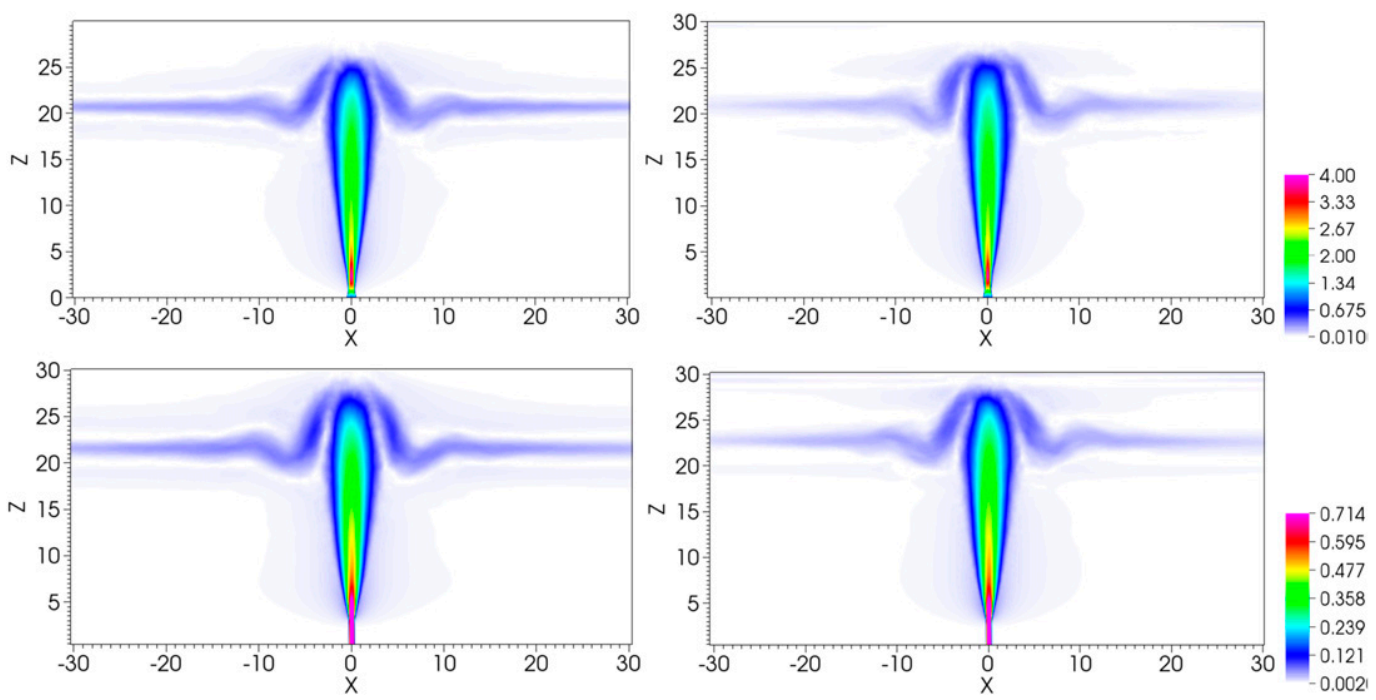

FIG. 4. Mean velocity magnitude in (left) winter and (right) summer stratification for (top) the lazy plume $\left(W_{01}=1.1 \mathrm{~m} \mathrm{~s}^{-1}\right.$ and $\left.D_{01}=20 \mathrm{~m}\right)$ and (bottom) the forced plume with increased subglacial discharge $\left(W_{03}=6.2 \mathrm{~m} \mathrm{~s}^{-1}\right.$ and $\left.D_{03}=10 \mathrm{~m}\right)$.

for the lazy and forced plumes as well as for the lazy plume in a homogeneous fluid (see appendix A for more detail). We assume that the plume velocity and density profiles are Gaussian, so that $b_{w}$ is the radius where the velocity falls $e$ times as compared to the maximal velocity in the corresponding horizontal cross section. In the range $7<z<18$, the data from all simulations follow the theoretical straight line with an entrainment coefficient $\alpha_{p g}=0.081$, a value typical for pure plumes in homogeneous fluids (Fischer et al. 1979; Ezzamel et al. 2015). Thus, the weak linear stratification up to the pycnocline does not influence turbulent entrainment. Note that we choose $z=18$ as the level where the plume enters the pycnocline, the location where the radius of the plumes in a stratified fluid starts to deviate from that in a homogeneous fluid (see Fig. 5).

The similar behavior of the forced and lazy plumes for the same basic discharge can be explained as follows: Given the same $Q_{0 i}=\pi W_{0 i} D_{0 i}^{2} / 4$ and the same initial density difference, both cases have the same buoyancy flux $F_{0 i}=g_{0}^{\prime} Q_{0 i}$, and the difference is therefore only in the initial momentum fluxes $M_{0 i}=\pi W_{0 i}^{2} D_{0 i}^{2} / 4$. In a homogeneous fluid, the buoyancy flux is conserved along the trajectory of a plume, and the MTT far-field theoretical solutions indicate that the momentum and volume flux are functions of the dimensional coordinate $z^{\prime}$ and the buoyancy flux $F_{0}$ (Morton et al. 1956):

$$
Q\left(z^{\prime}\right)=\left(\frac{5 \pi^{2} F_{0}}{8 \alpha_{p}}\right)^{1 / 3}\left[\frac{6 \alpha_{p}\left(z^{\prime}+z_{v}^{\prime}\right)}{5}\right]^{5 / 3}, \quad \text { and }
$$

$$
M\left(z^{\prime}\right)=\left(\frac{5 \sqrt{\pi} F_{0}}{8 \alpha_{p}}\right)^{2 / 3}\left[\frac{6 \alpha_{p}\left(z^{\prime}+z_{v}^{\prime}\right)}{5}\right]^{4 / 3} .
$$

Here, $\alpha_{p}$ is the entrainment coefficient under the assumption that the velocity profile is top hat $\left(\alpha_{p}=\sqrt{2} \alpha_{p g}\right)$, and we correct for nonzero mass and momentum fluxes of the source by introducing a virtual origin $z_{v}^{\prime}$. Solutions (7)-(8) are obtained for a point source of buoyancy (i.e., with no momentum or mass flux), and $z_{v}^{\prime}$ represents the vertical distance from the inflow where this virtual origin should be placed to produce a plume with the fluxes as those of the real plume in the far field (Hunt and Kaye 2001). Given the same $F_{0 i}$, we should asymptotically obtain the same solutions if the virtual origins are not far from each other, resulting in the same velocity $W\left(z^{\prime}\right)=M\left(z^{\prime}\right) / Q\left(z^{\prime}\right)$ for all the initial diameters, the only difference being the distance where the velocity approaches this asymptote.

The difference in the virtual origin position for the lazy and forced plumes can be estimated following Hunt and Kaye (2001). To use their correction for a virtual origin, we need the same expression for the source parameter $\Gamma_{0 i} \propto 1 / \mathrm{Fr}_{0 i}^{2}$ (see section 2). Having measured the plume entrainment coefficient $\alpha_{p}$, we use it to define $\Gamma_{0 i}$ as in Hunt and Kaye (2001):

$$
\Gamma_{0 i}=\frac{5}{8 \pi^{1 / 2} \alpha_{p}}\left(\frac{Q_{0 i}^{2} F_{0 i}}{M_{0 i}^{5 / 2}}\right) .
$$

This gives $\Gamma_{01}=12.16$ and $\Gamma_{02}=0.36$ for the lazy and forced plumes. Using the expression for the virtual 

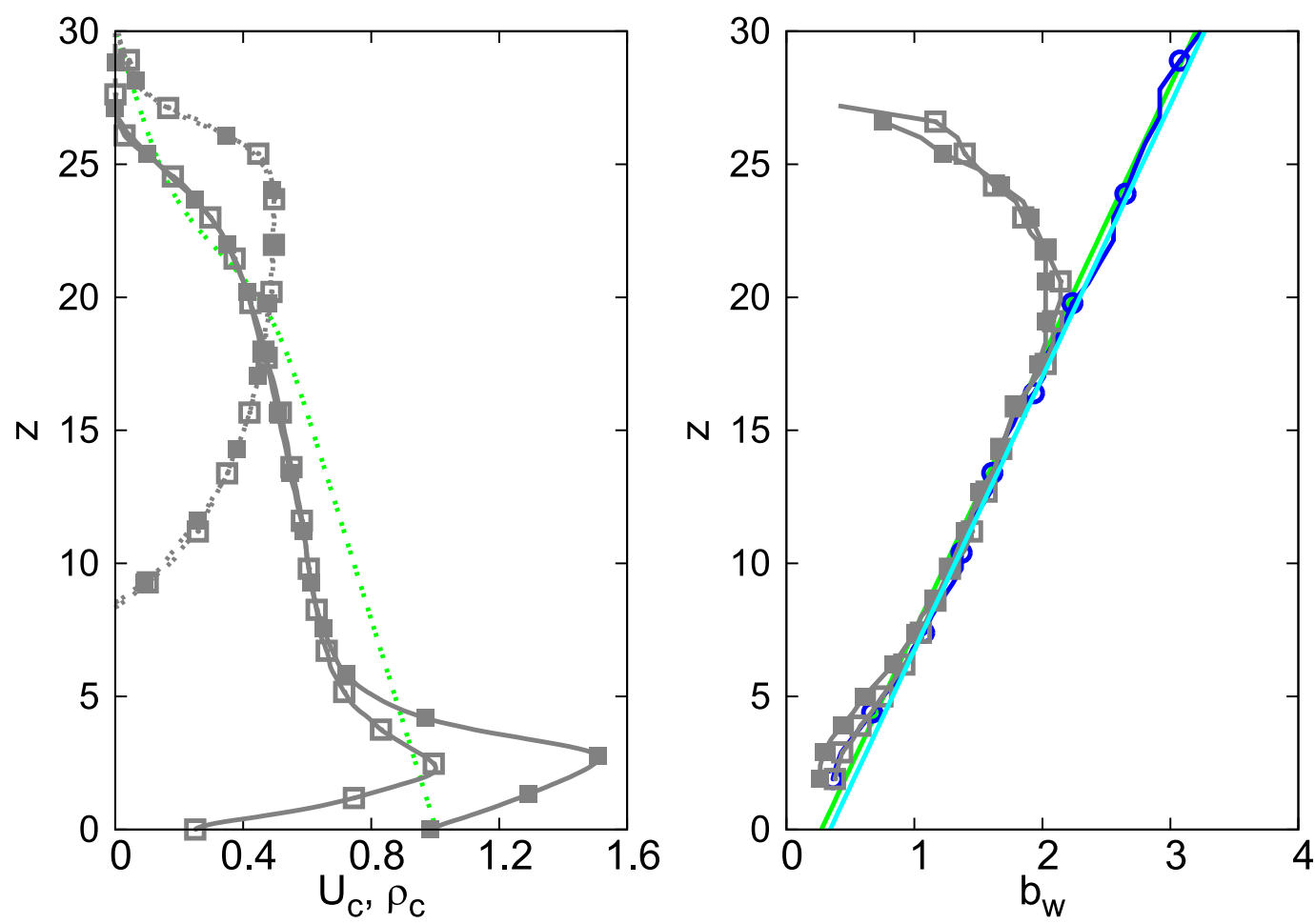

FIG. 5. (left) Mean vertical velocity (solid) and density (dashed) along the plume axis: lazy plume (open symbols) and forced plume (closed symbols) in winter stratification. Green curve shows the undisturbed density profile. Density along plume axis is shown only for $z>8$. (right) Variation of the plume radius with depth: plume in a homogeneous fluid (rounds), lazy plume (open squares), and forced plume (closed squares) for winter stratification. Green and cyan lines correspond to the theoretical MTT predictions with $\alpha_{p g}=0.081$ for lazy and forced plumes (in the literature $0.07<\alpha_{p g}<0.11$; see, e.g., Ezzamel et al. 2015).

origin in Hunt and Kaye (2001), we find that $z_{v 1}^{\prime} / L_{M 1}=-3.9$ for the lazy plume and $z_{v 2}^{\prime} / L_{M 2}=-1.6$ for the forced plume, where $L_{M i}$ is the momentum length $L_{M i}=\left[\left(5 M_{0 i}^{3 / 2}\right) /\left(9 \alpha_{p} \sqrt{\pi} F_{0 i}\right)\right]^{1 / 2}$, the distance after which the flow follows the asymptotic plume behavior given by (7)-(8). In our simulations, $L_{M i}$ is approximately $0.7 D_{0}$ for the lazy plume and $2 D_{0}$ for the forced plume. Thus, the difference between the virtual origins is comparatively small $z_{v 1}^{\prime}-z_{v 2}^{\prime} \approx 0.7 D_{0}$, and the theoretical predictions of the plume radius for the different virtual origins are close to each other (see Fig. 5) and within the scatter of the data.

We proceed to show that the lazy plume vertical velocity in the far field is not sensitive to the source diameter for a given fixed discharge. In the following analysis, we omit the indices, since the analysis is general and is not restricted to the parameters of our simulations. The analytical formula for the location of the virtual origin of turbulent lazy plumes (Hunt and Kaye 2001) can be expressed as a function of the source parameter $\Gamma$ and the momentum length $L_{M}$, valid for $0.5<\Gamma<\infty$ :

$$
\frac{z_{v}^{\prime}}{L_{M}}=-2.108 \Gamma^{3 / 10}(1-\delta),
$$

where $\delta$ varies from -0.071 to 0.147 when $\Gamma$ increases from 0.5 to $\infty$, so that the total change in $z_{v}^{\prime}$ caused by the coefficient $(1-\delta)$ is within $20 \%$. Given that $M_{0}$ is the only parameter that varies with the diameter, given a fixed discharge, we estimate the virtual origin location substituting $L_{M}$ and $\Gamma$ into (10):

$$
z_{v}^{\prime}=\frac{-2.108(1-\delta)}{3}\left(\frac{5}{2^{9 / 8} \sqrt{\pi} \alpha_{p}}\right)^{4 / 5}\left(\frac{Q_{0}^{3}}{F_{0}}\right)^{1 / 5} .
$$

We therefore obtain that $z_{v}^{\prime}$ depends on $M_{0}$ only through the coefficient $\delta$, and we can conclude that the position of the virtual origin may change only up to $20 \%$ for a wide range of diameters if the total discharge is fixed. Thus, the dependence of the plume behavior on the source diameter is weak, except for rather small sources with $\Gamma<0.5$ (our simulations show that, for the basic discharge, the difference between cases with $\Gamma=0.36$ and $\Gamma=12.16$ is still not significant). 

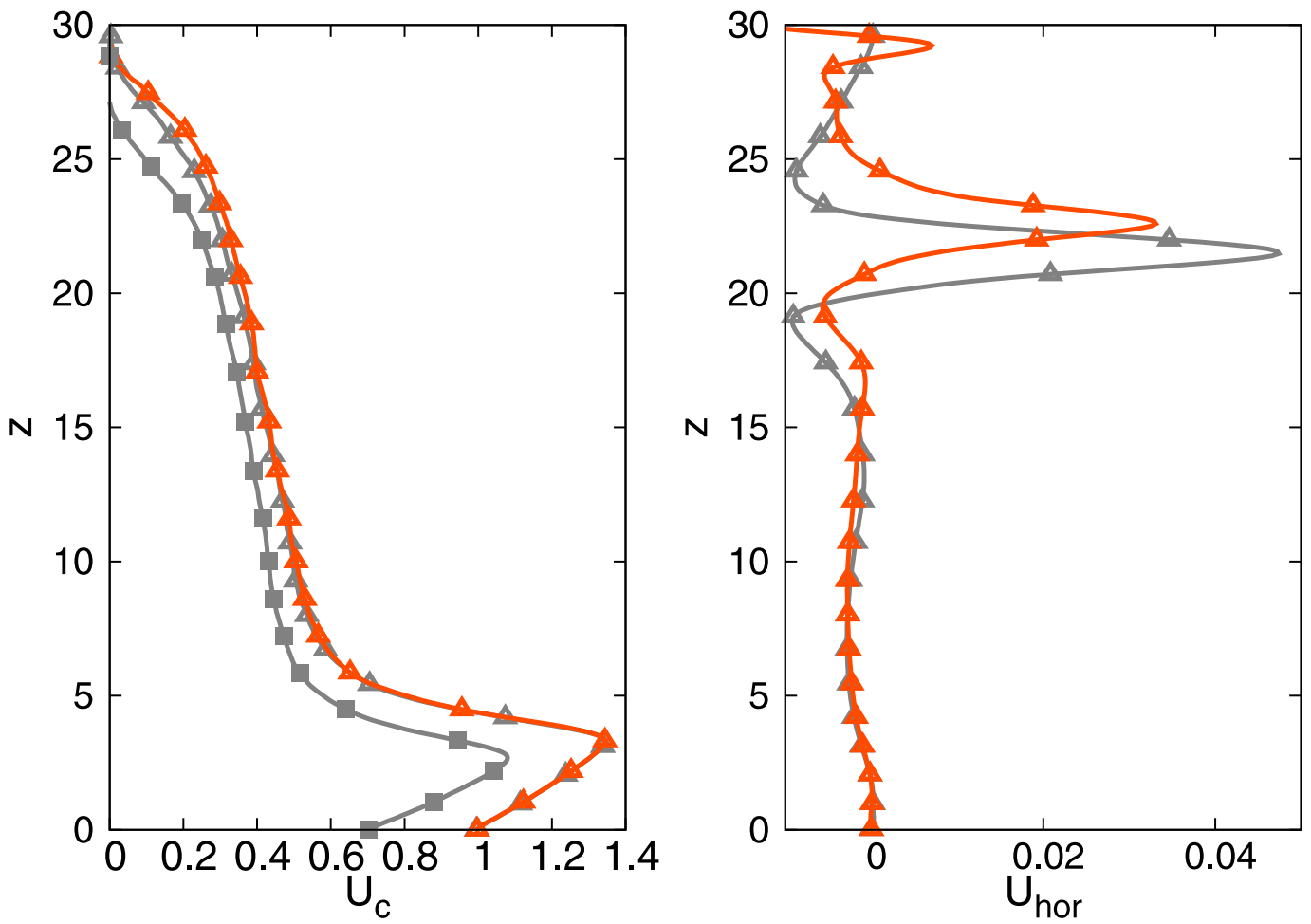

FIG. 6. (left) Mean vertical velocity along the plume axis. (right) Mean horizontal velocity at $r=20$. Forced plumes: basic discharge, winter stratification (squares), increased discharge, winter stratification (gray triangles), increased discharge, summer stratification (orange triangles).

For the basic discharge considered here, $z_{v}^{\prime} \approx$ $-3.3 D_{0}(1-\delta)$ for $0.5<\Gamma<\infty$. Increasing the diameter from $20 \mathrm{~m}$ to a larger value, with the same basic discharge, would change the virtual origin position by only $0.1 D_{0}$. In other words, the fluxes can be estimated by (7) and (8) and are almost the same for all reasonably large diameters.

Note that the momentum length is also important: the larger the momentum length, the longer the distance after which the plume can be described by solutions (7)-(8). Shabbir and George (1994) concluded, summarizing previous experiments, that the transition to the pure plume behavior occurs after $z^{\prime} \approx 5 L_{M}$. In their own experiments they observed this transition to occur for $6.5 L_{M}<z^{\prime}<16 L_{M}$. We emphasize here that at $z=7$ (i.e., at the distance where the mean centerline velocities of the lazy and forced plumes start to closely follow each other), the solution is not self-similar yet, as shown in appendix A, so it cannot be treated as a far-field asymptotic solution. For the lazy plume simulations, the transition to the behavior typical of a pure plume occurs after $z \approx 15$ (see appendix A). However, surprisingly, the difference in the initial conditions between the forced and lazy plumes becomes negligible much closer to the source, well before the plume approaches its asymptotic behavior.

Finally, we discuss the simulations with increased subglacial discharge: $W_{03}=6.2 \mathrm{~m} \mathrm{~s}^{-1}$ and $D_{03}=10 \mathrm{~m}$ in the winter and summer stratifications. The isocontours in the lower panel of Fig. 4 display the magnitude of the average velocity of this plume for both stratifications. The vertical profiles of the mean centerline velocities pertaining to these two cases are displayed in Fig. 6, together with the profile for the forced plume with the basic discharge. Again, the different curves follow nearly the same asymptotic behavior, with a difference of approximately 1.14 between the increased and basic discharge. For the increased discharge, $L_{M 3}=3 D_{0}$, and thus we can expect a transition to the pure plume behavior after $z^{\prime}=5 L_{M 3}$, that is, $z \approx 15$. However, the plume displays behavior close to a pure plume even in the transition region. Asymptotic theoretical solutions for a homogeneous fluid, (7) and (8), give a difference of 1.12 in the velocities (defined as $W=M / Q$ ) for these two cases, in close agreement with the results of our simulations. We therefore conclude that the plume behavior before the pycnocline is well described by the MTT theory for a homogeneous fluid, and the effect of the 

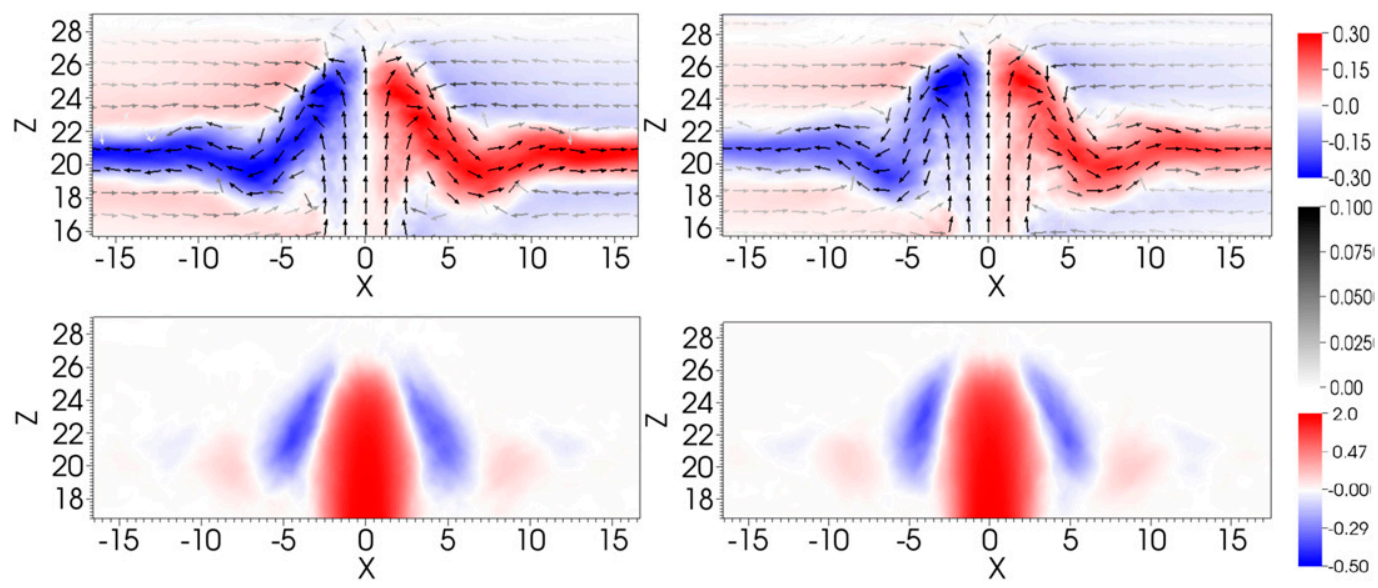

FIG. 7. The lazy plume in (left) winter and (right) summer. (top) Mean horizontal velocity (color scale) and vector velocity field (gray scale) and (bottom) mean vertical velocity (color scale). The length of vectors in the upper panels is fixed, and the difference in velocity magnitude is illustrated using the color scale.

slightly linear stratification on the turbulent entrainment in the lower layer is negligible.

Note, that a 1.5 times increase in subglacial discharge causes a rather modest increase in the plume velocity, only by $\sim 10 \%-15 \%$. This is due to the weak dependence of the plume velocity on the source volume flux $W \sim Q_{0}^{1 / 3}$, as follows from MTT theory. Given that the penetration height, melting rates, and frequency of plume oscillations depend on the plume velocity, this leads to an important conclusion that the plume dynamics are not very sensitive to the subglacial discharge magnitude, unless its deviations from the mean value considered allows the plume to reach the free surface (for very large discharges) or the buoyancy forcing caused by melting becomes important (for very low discharges).

\section{b. Turbulent entrainment}

We proceed by estimating the stratified turbulent entrainment as in Ezhova et al. (2016). A movie illustrating the density evolution of the lazy turbulent plume in the summer stratification (case 1 in Table 1) can be found in the supplemental information. The visualizations of the mean velocities of the forced and lazy plumes in Figs. 4 and 7 show that the plume penetrating into the pycnocline forms a domelike structure. The entrainment coefficient can be defined by the formula $E=Q_{e} / Q_{\text {in }}$ (Shrinivas and Hunt 2014), where $Q_{e}$ is the volume flux of the fluid entrained by the top of the plume (i.e., by the dome) and $Q_{\text {in }}$ is the volume flux of the plume at the entrance to the pycnocline. It is important to note that $E$ concerns only the entrainment occurring through the surface of the dome and is different from the classical entrainment coefficient $\alpha_{p}$, which is associated with the entrainment along the conical side of the rising vertical plume. The inflow volume flux $Q_{\text {in }}$ is computed at $z=18$, at the entrance to the pycnocline, as discussed in the previous section. Given that the characteristics of the plumes (velocity and diameter) are similar at this level for the same discharge, the plume volume flux $Q_{\text {in }}$ is the same, and the differences we will report are due to the fluid entrained through the dome surface $Q_{e}$. As discussed previously for a jet penetrating in a thick thermocline (Ezhova et al. 2016), entrainment occurs not homogeneously over the surface of the dome but preferably through the sides (see Fig. 7, where the horizontal flows toward the plume are displayed); this appears reasonable in a stably stratified fluid where the vertical turbulent transfer is decreased. As a result of the calculations, we obtain that the entrainment coefficient $E=0.61$ in winter. In summer, instead, the stratified entrainment is significantly decreased to $E=0.43$.

To compare the results with those of a jet penetrating in a two-layer stratified fluid (Shrinivas and Hunt 2014; Ezhova et al. 2016), we estimate the Froude number at the entrance of the pycnocline as $\operatorname{Fr}_{p}=W_{p} / \sqrt{g_{p}^{\prime} R_{p}}$. Here, $W_{p}$ and $R_{p}$ are the dimensional mean velocity and radius of the plumes at $z=18$ (these parameters are the same for the lazy and forced plumes; see Fig. 3). The mean velocity is estimated as $W_{p}=W_{0 i} U_{c} / 2$, while $R_{p}$ is the radius where the velocity equals to $U_{c} / 2$, for these cases $W_{p} \approx 1 \mathrm{~m} \mathrm{~s}^{-1}$ and $R_{p} \approx 32 \mathrm{~m}$. The reduced gravity is calculated using the density values in the plume at $z=18\left(\rho_{z=18}=\int_{0}^{\infty} \rho U_{z=18} r d r / \int_{0}^{\infty} U_{z=18} r d r=0.52\right)$ and the mean density of the ambient fluid from $z=18$ to the penetration depth. The latter is estimated to be approximately $\rho=0.29$, resulting in a dimensional value of 
the reduced gravity $g_{p}^{\prime}=0.0082 \mathrm{~m} \mathrm{~s}^{-2}$; the Froude number obtained with these parameters is thus $\operatorname{Fr}_{p} \approx 1.95$. For this Froude number the entrainment coefficient for a jet in a two-layer stratification $E \approx 0.7$ (Shrinivas and Hunt 2014; Ezhova et al. 2016), higher than obtained from the present simulations. Note, however, that if we make estimates based on the density of the ambient fluid averaged over the height of the dome above the horizontal gravity current, where entrainment is occurring, we obtain $\rho=0.16$ in winter and $\rho=0.14$ in summer with $\operatorname{Fr}_{p} \approx 1.58$, which for a jet gives $E \approx 0.56$, in closer agreement with the present estimates in winter when the stratification is closer to two layers. The fact that the Froude number, calculated with the density averaged from the pycnocline entrance to the plume top (as if the pycnocline thickness is negligible), results in a larger deviation of the entrainment coefficient from the theory of Shrinivas and Hunt (2014) emphasizes the importance of the finite thickness of the pycnocline for the estimates of entrainment in winter stratifications. In summer, the pycnocline is very thick, and comparison with the twolayer theory is not justified.

From the data in Fig. 7 (upper panel), we deduce that the reduced entrainment observed in summer results both from the decrease in the surface of the dome, formed when the plume penetrates into the pycnocline and from the smaller rates of entrainment, possibly because of the larger ambient density gradient dampening turbulent entrainment (note that the difference both in entrainment velocities and dome heights is not large, since the difference in stratifications is important only near the surface and the plume does not penetrate to the surface). One can therefore suggest the following explanation for the difference in entrainment.

The turbulent entrainment is proportional to the entrainment rate multiplied by the surface of the dome. The latter is estimated roughly as the half-surface of a prolate spheroid:

$$
S_{\text {prol }}=\pi a\left[a+\frac{b^{2}}{\sqrt{b^{2}-a^{2}}} \arcsin \left(\frac{\sqrt{b^{2}-a^{2}}}{b}\right)\right] .
$$

For lazy and forced plumes in winter, the semiminor axis is $a=3.3$ and semimajor axis is $b=4.5$, while in summer $a=3.2$ and $b=3.8$, resulting in $S_{\mathrm{prol}}^{\mathrm{win}}=86$ and $S_{\text {prol }}^{\text {sum }}=72$. The entrainment rate is estimated as the velocity magnitude of the ambient fluid on the surface of the dome averaged over the height of the dome, giving $u_{e}^{\text {win }}=0.073$ in winter and $u_{e}^{\text {sum }}=0.064$ in summer. Thus, the ratio of the entrainment coefficients $\quad E^{\text {win }} / E^{\text {sum }}=\left(S_{\text {prol }}^{\text {win }} u_{e}^{\text {win }}\right) /\left(S_{\text {prol }}^{\text {sum }} u_{e}^{\text {sum }}\right) \approx 1.36$ is in a good agreement with the $30 \%$ reduction in the entrainment coefficient we obtained from the direct calculations.

The size of the dome surface is connected with the upper boundary of the horizontal flow (Fig. 7, upper panel). As will be discussed later, the difference in this level between summer and winter can be explained by the difference in ambient stratifications. While the density distribution in the downflow is approximately similar, the ambient stratification arrests the downflow at a higher level in summer. This is because the lighter part of the fluid inside the downflow becomes neutrally buoyant earlier in summer than in winter.

We estimate the turbulent entrainment at the top of the plume for the simulations having an increased discharge (cases 4 and 5, Table 1). The estimates are $E=0.75$ for the winter conditions and $E=0.38$ for the summer stratification. The parameters required for the calculations of $\mathrm{Fr}_{p}$, the mean velocity and radius, are $W_{p} \approx 1.15 \mathrm{~m} \mathrm{~s}^{-1}$ and $R_{p} \approx 33 \mathrm{~m}$. The Froude number based on the difference between the plume density at $z=18$ and the density averaged over the distance from the pycnocline entrance to the maximum penetration point $z \approx 28.5$ is $\operatorname{Fr}_{p} \approx 2.3$. The theoretical estimate of the entrainment coefficient for a turbulent jet in a two-layer stratification with $\operatorname{Fr}_{p}=2.3$ is $E=0.85$ (Shrinivas and Hunt 2014), again larger than the values from the present simulations. However, estimates based on the density averaged over the dome height, as for the basic discharge, give $\mathrm{Fr}_{p}=1.9$ in winter and $\mathrm{Fr}_{p}=1.58$ in summer. The corresponding entrainment coefficient is $E \approx 0.7$ in winter and $E \approx 0.56$ in summer. Thus, again the value of the entrainment coefficient in winter is close to the theoretical value obtained for a jet in a two-layer stratification, while in summer the entrainment is lower. The corresponding horizontal velocity for the simulations with increased discharge is illustrated in Fig. 6. The horizontal flow in summer is slower and propagates significantly higher in the water column, which results in a reduced surface of the dome and a consequent reduction in entrainment.

To estimate the energy exchange between the plume and the surrounding fluid, we have calculated the energy fluxes in several cross sections inside the plume top. The total energy flux is $\varepsilon_{\text {tot }}=\int\left\{\left(U^{2} / 2\right)+\left[\left(\rho_{p l}-\rho_{s}^{\prime}\right)\right.\right.$ $\left.\left.\left(z-z_{\text {neut }}\right) / \mathrm{Fr}_{i}^{2}\right]\right\} U d S$, where $\rho_{p l}$ is the density of a liquid parcel inside the plume, $\rho_{s}^{\prime}$ is the ambient density at the same level, $z$ is the current vertical coordinate of the liquid parcel inside the plume, and $z_{\text {neut }}$ is the level of neutral buoyancy for this parcel.

Integration is performed in the horizontal cross section at $z=18$ and then over the conical and cylindrical surfaces crossing the plane $y=0$ along the lines shown in Fig. 8. The cross sections are numbered so that the thin, 

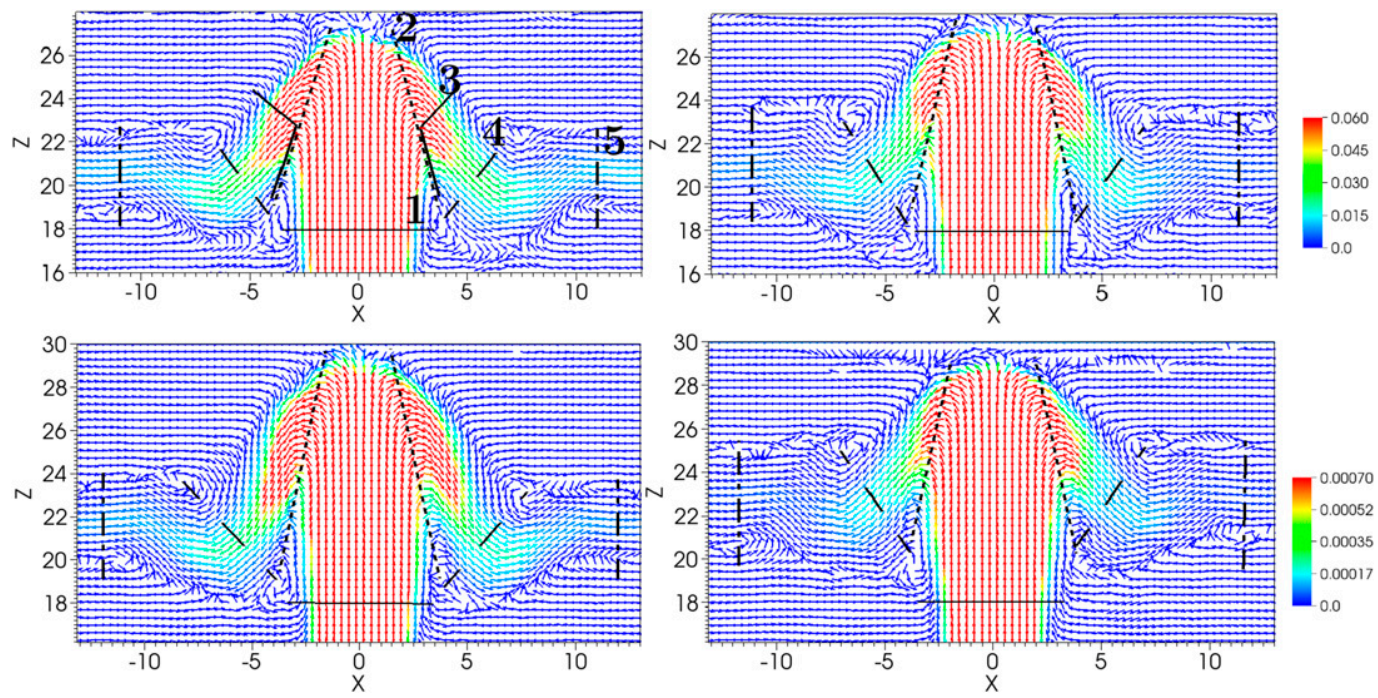

FIG. 8. Vector energy flux fields (color scale) and sections across the plume used to calculate the energy flux at different locations. The size of the arrows is fixed. (top) Basic discharge and (bottom) increased discharge. (left) Winter and (right) summer.

solid surface is number 1 , dotted is number 2 , thick solid is number 3 , dashed is number 4 , and dashed-dotted is number 5. Cross-section 3 has been defined only for case 1 (Table 1), since its position, intended to be approximately in the middle of the downflow, is a bit arbitrary. The same numeration pertains to all the panels in Fig. 8 . These surfaces are chosen nearly normal to the flow. After the plume reaches its maximum height, the energy flux in the plume decreases drastically. Considering the basic discharge simulations, in winter (summer), $86 \%$ ( $76 \%)$ of the total energy flux of the incoming plume is available in the ascending plume, and the plume loses $70 \%(63 \%)$ of the initial energy flux during its descending motion, most probably to entrainment and mixing (Fig. 9). This argument is supported by Fig. 10, displaying the mean density and approximate plume boundaries shown with the contour lines of the horizontal (dotted) and vertical (dashed) velocities. The density in the ascending plume is always different to the density in the downflow, which is lighter. Thus, the potential energy in the downflow decreases. Note that previous experimental estimates of the energy expended on entrainment by the plume top in a two-layer stratified fluid with a sharp density interface (Cardoso and Woods 1993 ) provided values around $50 \%$, but in our case the plume penetrates further in the upper layer because the pycnocline has a finite thickness. For the increased discharge the energy fluxes through the respective cross sections are nearly the same as for the basic discharge.

Contours of horizontal velocity in Fig. 10, adjacent to the dome surface, indicate the level where entrainment by the plume top is most effective. It can be seen that in summer, both for basic and increased discharge, the plume entrains lighter fluid. Thus, in winter, the density difference between the plume and entrained fluid is smaller, but the amount of the entrained fluid is larger (entrainment coefficient $E$ is larger in winter). In summer, the plume entrains a smaller amount of lighter fluid, but the fraction of energy expended by the plume to entrainment and mixing is nearly similar in all cases $(60 \%-70 \%$ of the inflow energy). As the plume intrudes

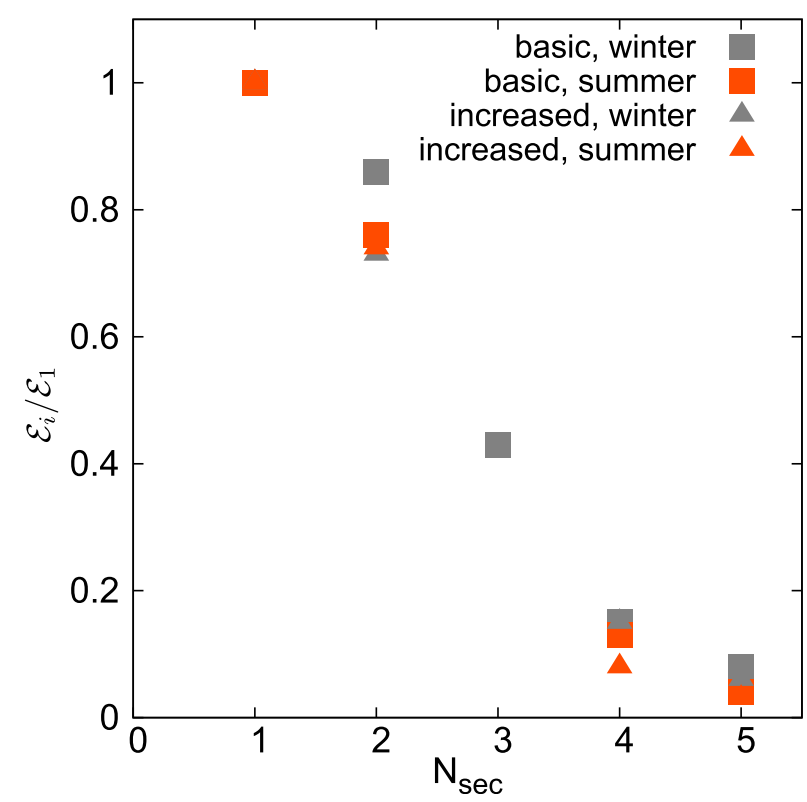

FIG. 9. Fraction of incoming energy flux vs cross-section number. 

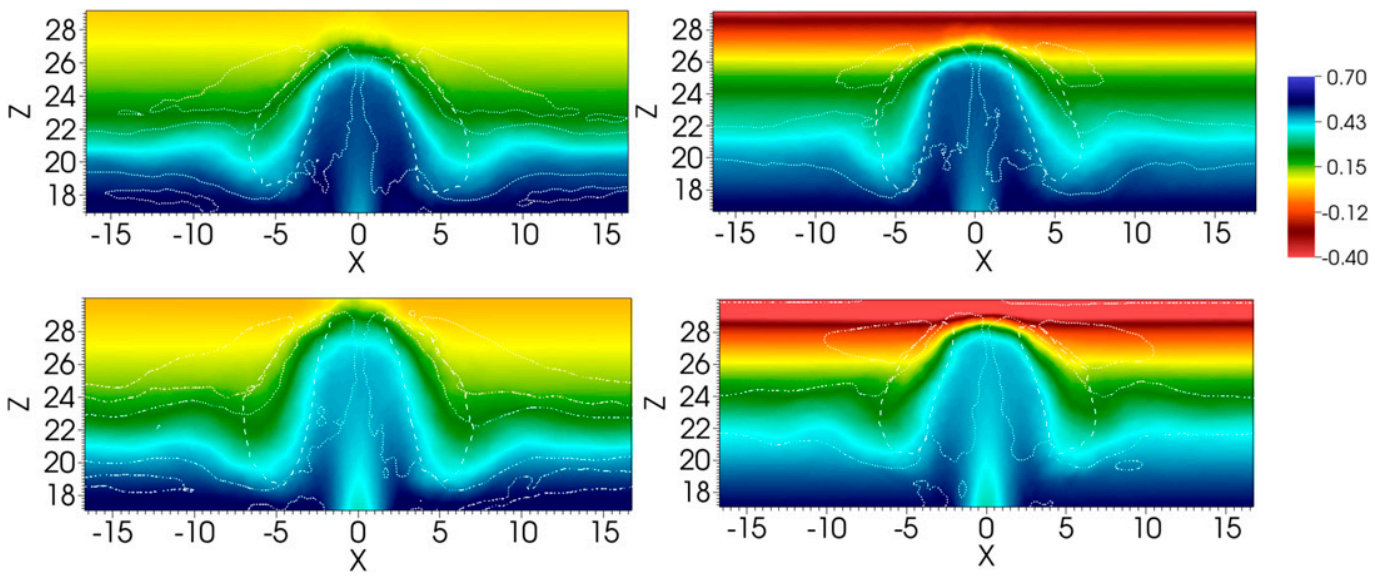

FIG. 10. As in Fig. 8, but for the mean density. Dashed lines represent contours of vertical velocity (upper panel: -0.06 , lower panel: -0.011 ); dotted lines represent contours of horizontal velocity (upper panel: -0.05 and -0.05 , lower panel: -0.0089 and 0.0089 ).

to form a horizontal density current, the energy loss is small and only $\sim 7 \%-10 \%$ of the initial energy flux.

Finally, we comment on the expected effect of entrainment by the plume top on the melting rate. The fountain generated by the plume penetrating through the pycnocline is more than $100 \mathrm{~m}$ high for the discharges considered here, so that the contribution of the downflow to melting, which is often disregarded, can be significant. Note that, of the two scalars that define stratification, that is, temperature and salinity, it is salinity that contributes most to the density change and thus has a strong impact on the plume dynamics. In Greenland fjords cold waters are located above warm waters, and already at $z=22$ the temperature has decreased by several degrees, compared to the temperature below, which means that the plume top entrains cold water. In winter, the salinity of the Sermilik Fjord decreases toward the surface from 34.8 to $33 \mathrm{~g} \mathrm{~kg}^{-1}$, the temperature decreases from $4^{\circ}$ to $-2^{\circ} \mathrm{C}$, and the mean downflow temperature for the basic discharge is around $1.7^{\circ} \mathrm{C}$. In summer, salinity decreases smoothly from 35 to $33 \mathrm{~g} \mathrm{~kg}^{-1}$, while temperature jumps from $3^{\circ}$ to $0.5^{\circ} \mathrm{C}$, giving as temperature of the downflow around $2.2^{\circ} \mathrm{C}$. The effect of the temperature on the melting rate can be estimated as $\mathrm{MR} \sim C_{D} U\left(T-T_{L}\right.$ ) (Holland and Jenkins 1999), where $T_{L}$ is the freezing point temperature of freshwater, $T_{L}=-1.2^{\circ} \mathrm{C}$ at $z=22$, and $C_{D}$ is the drag coefficient. Thus, in stratifications typical of winter (entrainment coefficients of order unity, low temperatures in the upper layer) melting rates in the downflow are expected to decrease by $\sim 50 \%$ as compared to the melting rates in the upward flow for the same velocities, while the downflow contribution will be more significant in summer (decreased entrainment and higher temperatures of entrained water). Furthermore, as can be seen from Fig. 4, the velocity in the downflow is decreased by a factor of $2-3$, as compared to the ascending plume at the same level for both stratifications; thus, the melting associated with the downflow is expected to decrease proportionally.

\section{c. Internal waves}

We have shown (Ezhova et al. 2016) that vertical turbulent jets in a two-layer stratified fluid can oscillate and generate internal waves with an energy of about $3 \%-5 \%$ of the jet energy at five jet diameters (taken at the entrance to the pycnocline) away from the jet axis. The Froude number at the pycnocline entrance investigated in this previous study was in the range 0.6-1.9. The Froude number of the plumes considered here (taken at $z=18$ ) is about 1.6 for the basic discharge and 1.9 for the increased discharge. A jet with this Froude number in a two-layer stratification shows strong oscillations of the jet top at two well-pronounced frequencies. The spectra of the plume oscillations in the more complicated stratifications investigated in the present study are, however, different; they are broader and noisier, though two pronounced peaks can also be seen in some of the spectra. Here, as in Ezhova et al. (2016), we use power spectra of isopycnal displacements to illustrate plume top oscillations and internal waves.

The summer stratification is characterized by a thick pycnocline with the sharpest density gradient in the immediate vicinity of the surface (the layer of thickness $\sim D_{0}$ under the surface, which is referred to hereinafter as the subsurface layer). Thus, one may expect that the oscillations of the plume will generate gravity waves characterized by different frequencies at the different $z$ levels. Figure 11 shows the isopycnals at a distance of $r=20$ from the center of the plume; the oscillations close to 


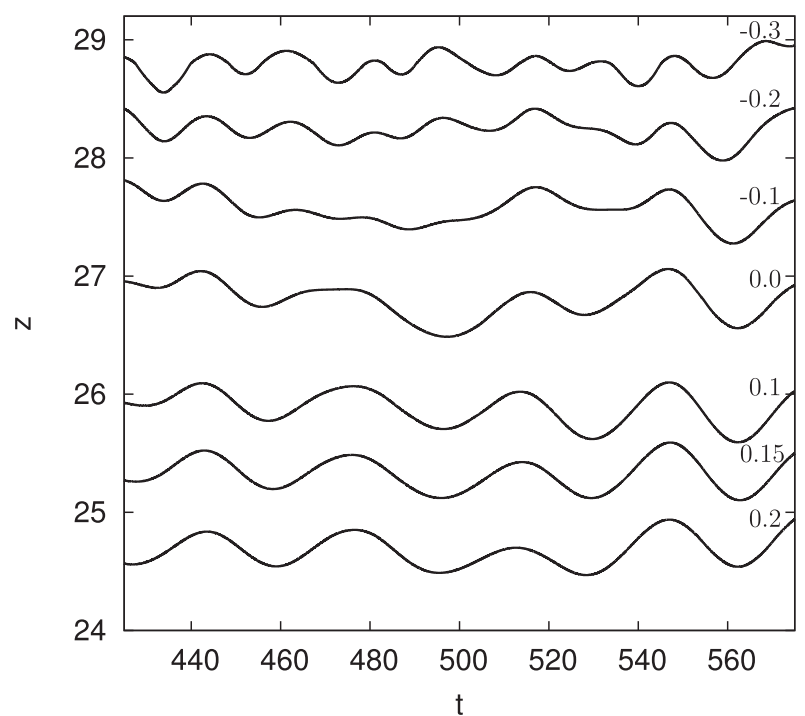

FIG. 11. Isopycnals at a distance $r=20$ from the center of the plume in the summer stratification for the basic discharge. The labels denote the corresponding density.

the surface clearly have higher frequencies. The spectra of plume oscillations and internal waves extracted at two different levels are reported in Fig. 12. The first level is located close to the surface $(z \approx 29 ; \rho=-0.3)$, and the second is located just above the horizontal gravity current $(z \approx 23.5 ; \rho=0.3)$. The isopycnals characterizing the plume oscillations correspond to $\rho=0.15$ and are taken at $r=0$ and four points on the circle of radius $r=1.5$ (the density value $\rho=0.15$ on the axis of the plume is reached at $z=27$ where the mean vertical centerline velocity $U_{c}$ falls to zero, and thus it is taken to be the characteristic density of the plume top). The resulting spectrum of plume oscillations is averaged over five spectra of isopycnal displacements. The difference between the spectra in Fig. 12 (upper panel) can be explained as follows: In the subsurface layer (i.e., $z \approx 29$ ), the local buoyancy frequency is higher than the frequencies of the energy-containing harmonics in the spectrum of plume oscillations. Thus, the spectrum of internal waves measured in the subsurface layer contains all the same harmonics. At the same time, the local buoyancy frequency deeper in the water column is lower (i.e., $z \approx 23.5$ ), and it cuts off part of the spectrum.

In winter and summer, the stratification profiles are close to each other in the proximity of the horizontal gravity current (at $z \approx 23-24$ ), and at that level the corresponding spectra of internal waves look similar, with one pronounced peak at the same frequency (cf. top left panel with bottom panel in Fig. 12).

Finally, we display in Fig. 13 the spectra of the forced plume oscillations and corresponding internal waves in the winter stratification for the basic and increased discharge (all the spectra are shown in their nondimensional variables). Again, the spectra representing the plume oscillations are noisy, whereas the spectra of internal waves have only one peak close to the maximal buoyancy frequency. In dimensional variables, we get the following estimates for the periods of the internal waves $\left(T_{\mathrm{IW}}\right): 1$ ) winter stratification, lazy plume $-T_{\mathrm{IW}}=13 \mathrm{~min}$; 2) winter stratification, forced plume, basic discharge $T_{\mathrm{IW}}=12.5 \mathrm{~min}$; and 3 ) winter stratification, forced plume, increased discharge $-T_{\mathrm{IW}}=13.5 \mathrm{~min}$.

Thus, in winter, internal waves are characterized by a period $T_{\mathrm{IW}} \approx 13 \mathrm{~min}$, corresponding to a frequency $\approx 0.7 N_{\text {max }}$ ( $N_{\text {max }}$ is the maximum buoyancy frequency), independently of the quantity of discharge and diameter of the source. This is in agreement with the results of Ansong and Sutherland (2010) on the internal waves generated by buoyant plumes in a stratification having a uniformly stratified layer above a homogeneous layer. These authors have found that there is no direct relation between the frequency of oscillations of the plume and the characteristic frequency of internal waves, which is always between $0.5 \mathrm{~N}$ and $0.8 \mathrm{~N}$ ( $N$ is the buoyancy frequency, constant for a linear stratification). However, our results for jets in a stratification having two layers separated by a pycnocline do show a correlation between the frequencies of the jet oscillations and internal waves (Ezhova et al. 2016). A possible explanation is that the waves generated by the plume oscillating in a stratified fluid interact with the oscillations of the plume, and this is why the spectra of the plume oscillations are noisier than the spectra of jet oscillations. When a jet penetrates through the pycnocline between the layers, the jet top oscillates in a homogeneous upper layer and thus is less influenced by the motion of the water column.

In summer, the internal waves with a wide, noisy spectrum propagate close to the surface. Internal waves in the subsurface layer may serve as an indicator of a submerged plume, which is relevant for remote detection of subglacial discharges. First, the proximity of the pycnocline to the surface increases the possibility for internal waves to be detected by remote sensing. Second, internal waves may mix the subsurface layer, and a reduction of the strong temperature and salinity gradient near the surface, typical of a summer stratification, may also point to the presence of oscillating plumes in the water column.

\section{Conclusions}

We have presented the results of numerical simulations of a vertical turbulent buoyant plume in a stratified fluid, with the stratification taken to be that characteristic of summer and winter conditions in Greenland 

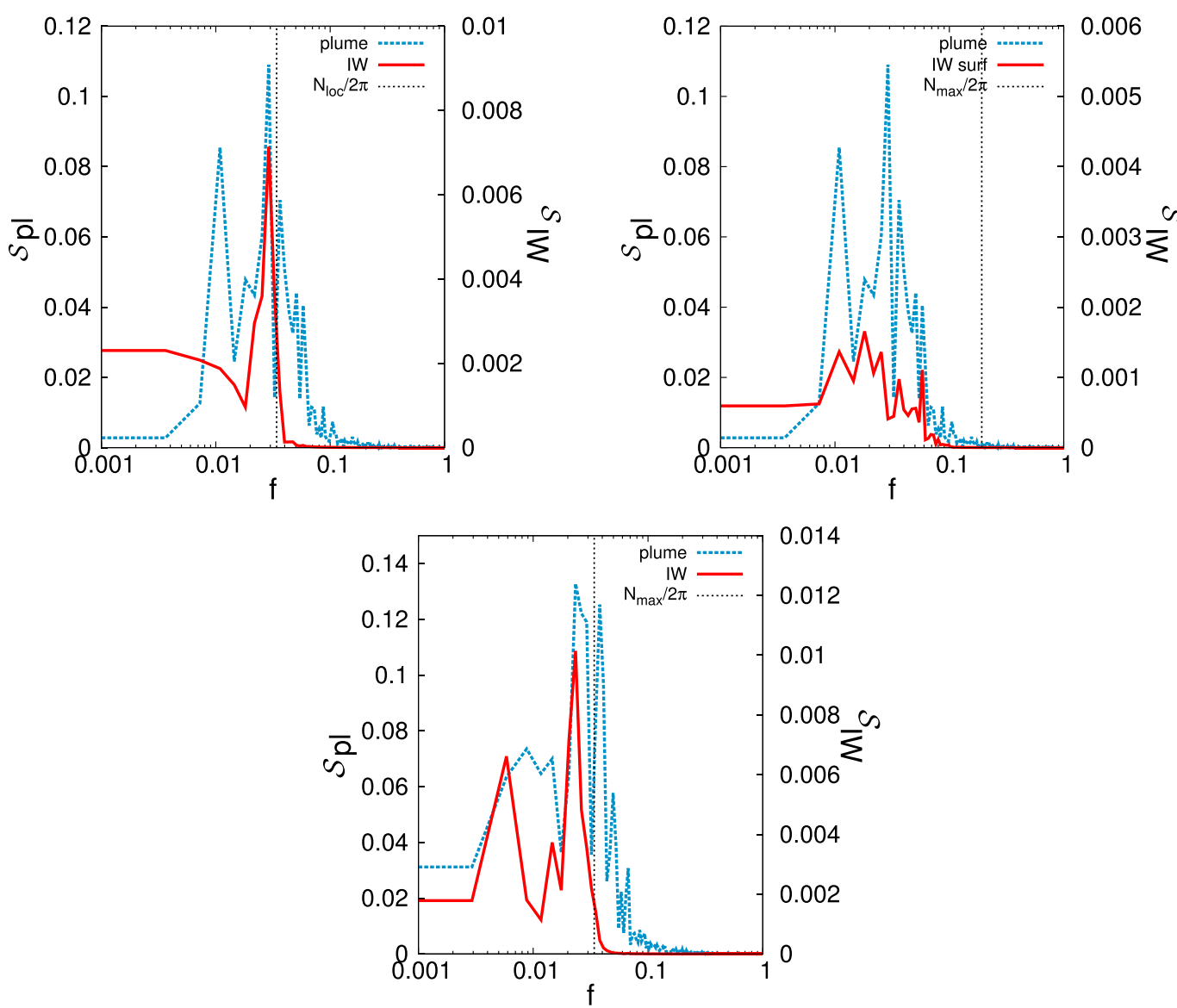

FIG. 12. (top) Spectra of lazy plume oscillations and internal waves in the summer stratification. (left) Internal wave corresponding to the isopycnal at $z \approx 23.5$, and (right) internal wave corresponding to the isopycnal at $z \approx 29$. (bottom) Spectra of lazy plume oscillations and internal waves in the winter stratification $(z \approx 23.5)$.

fjords. The total discharge generating the plume is chosen to model the subglacial discharge of a typical marine-terminating glacier, and two realistic values of the source diameter are considered. Different source diameters for the same amount of discharge imply different initial momentum fluxes, and the resulting plumes are therefore classified as lazy and forced. Given the different initial conditions, one might expect a difference in turbulent entrainment and different dynamics of the flow.

However, the simulations reveal that the difference between the different cases is small, and the flows display similar dynamics already at $7 D_{0}$ above the source ( $D_{0}$ being the largest source diameter), independently of the initial diameter. This is in agreement with the theoretical predictions (based on MTT theory) for pure plumes in a homogeneous fluid having the same buoyancy flux. Moreover, we show that the influence of the weak linear stratification, characteristic of some of the Greenland fjords, on the turbulent entrainment is negligible up to the pycnocline, that is, the entrainment coefficient of the plume in the weakly stratified fluid is the same as the one for the same plume in a homogeneous fluid. This means that the entrainment and plume dynamics in this lower, weakly stratified layer can be modeled using MTT theory, strictly valid for a homogeneous ambient fluid (Slater et al. 2015; Mankoff et al. 2016). This result is confirmed by additional simulations with increased discharge; the difference in velocity at the pycnocline between plumes with basic and increased discharge is well predicted by the MTT theory. This simplifies substantially the modeling and in particular allows the use of the analytical self-similar solutions from MTT.

The seasonal variation in the stratification is significant only in the upper 200-m layer. This has a large impact on the turbulent entrainment occurring near the top of the plume. In winter, the entrainment coefficient can be estimated using theoretical expressions for a twolayer stratification by Shrinivas and Hunt (2014), if we 

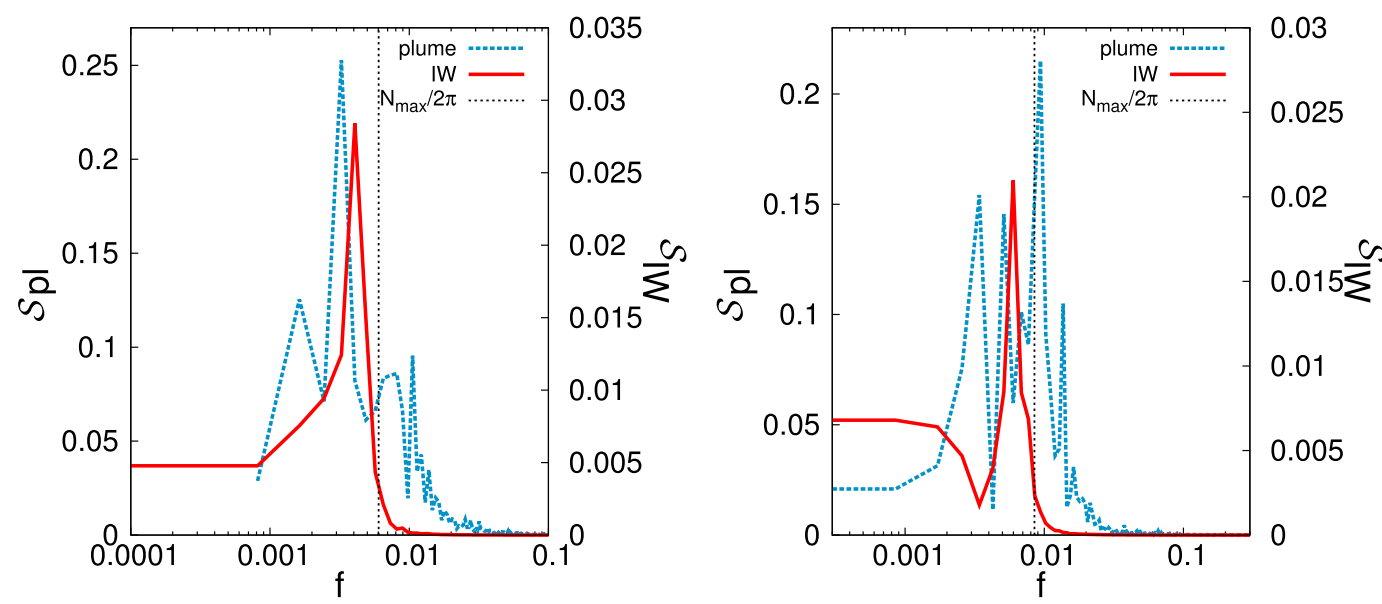

FIG. 13. Spectra of forced plume oscillations and internal waves in the winter stratification: $z \approx 24$. (left) Basic discharge and (right) increased discharge.

calculate the inflow flux at the pycnocline entrance (where the inflow is not yet affected by the counterflow), while the density of the upper layer should be taken as the average from the level above the gravity current until the penetration height, given that this is the region where entrainment occurs. In summer, however, the entrainment is reduced. This can be explained by the difference in stratifications; in summer, the stratification arrests earlier the downflow in the fountain.

In addition, we have investigated the generation of internal waves by the plume for the different stratifications and discharges considered. The spectra of the plume oscillations in these realistic stratifications are noisier than the spectra of turbulent jet oscillations in a two-layer stratification, either because of the feedback imposed by the oscillating stratified water column on the plume or because of the proximity of the top of the plume to the surface. In winter, the plume generates weak internal waves with a pronounced spectral peak at frequency close to $0.7 N_{\text {max }}$. In summer, instead, the plume generates internal waves with a peak at $0.7 N_{\text {loc }}$, where $N_{\text {loc }}$ is the local buoyancy frequency in the water column close to the entrance to the pycnocline. This is in agreement with available experimental results on the generation of internal waves in a linear stratification above a homogeneous layer (Ansong and Sutherland 2010).

In summer, additionally, the plume oscillations generate internal waves with a broad and noisy spectrum in the subsurface layer, characterized by a strong density gradient. These waves may mix the subsurface layer even if the plume does not penetrate to the surface.

There are several implications of the results found with this idealized model of subglacial discharge in Greenland fjords for the real geophysical setting motivating this study. The fact that the plume behavior and dynamics are not affected, to first order, by the source's diameter suggest that the exact knowledge of this variable is not essential. This is encouraging, given the fact that the exact location, distribution, and size of the subglacial discharge openings are not known in the field and are very difficult quantities to measure. Furthermore, the results suggest that in both seasons the subglacial discharge plume is not expected to penetrate all the way to the free surface. Instead the buoyant plume should intrude into the fjord along the interface between the Atlantic and polar layers, in agreement with the recent findings of Carroll et al. (2016) and what was previously suggested by field observations (Straneo et al. 2011) and numerical simulations (e.g., Sciascia et al. 2013). The results obtained with respect to the internal wave generation are an interesting aspect that, to our knowledge, has not been the focus of observational programs. It would be interesting to verify if the internal wave peak at $0.7 \mathrm{~N}$ observed both in summer and in winter is a signal detectable in available or future field observations. In summer, if this phenomenon occurs in the field, it may provide a mechanism to further mix waters near the surface and may become an indicator of a submerged plume if detected by remote sensing. It is important to note that we expect the results of this study to pertain mainly to summer conditions, as in winter subglacial discharge is strongly reduced.

Acknowledgments. We thank three anonymous reviewers for their comments improving the clarity of the discussions in the manuscript. This work was supported by Linné FLOW Centre at KTH and the Academy of Finland Centre of Excellence program (Grant 307331) (E. E.) and VR Swedish Research 

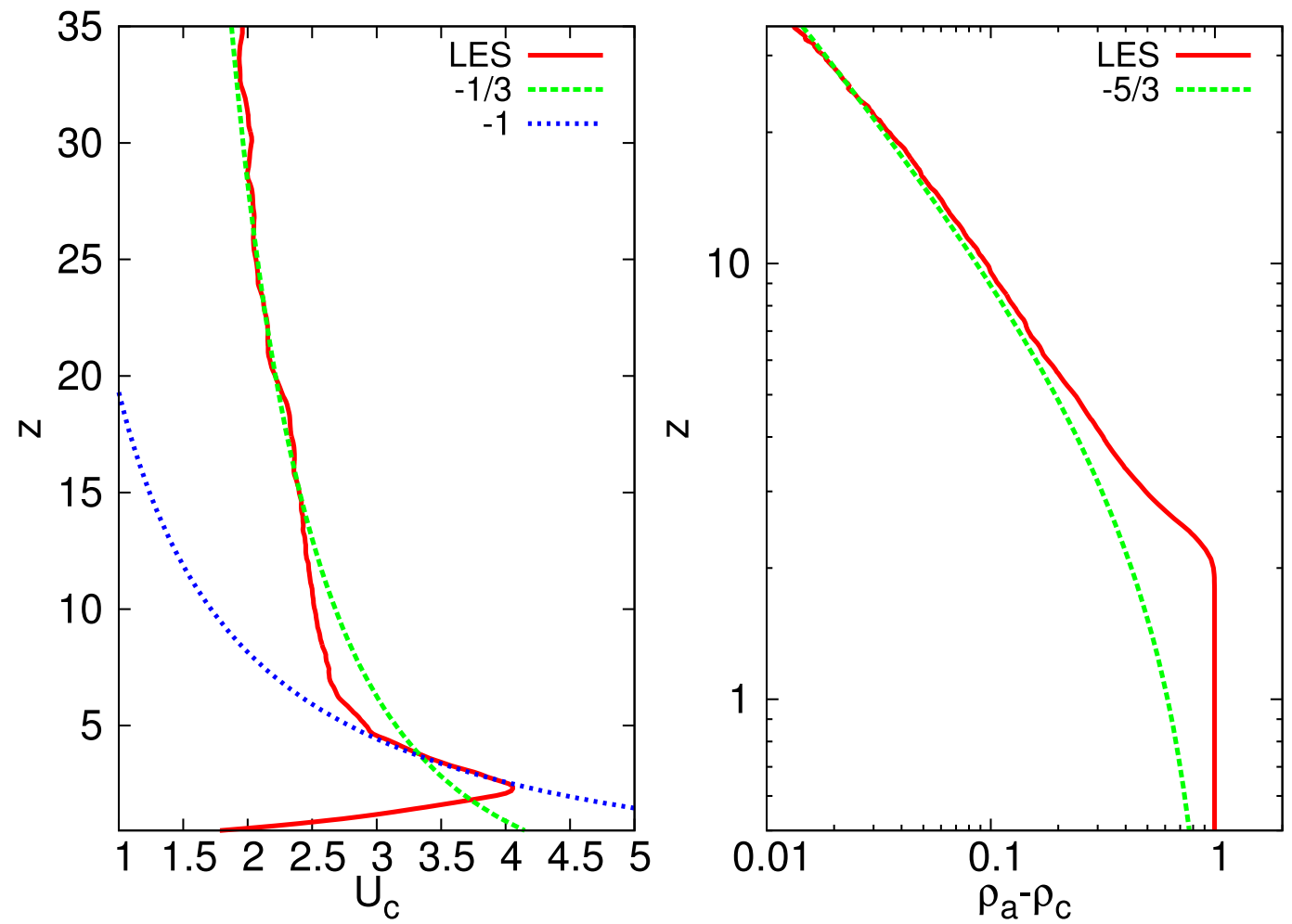

FIG. A1. (left) Mean vertical velocity along the plume axis. (right) Mean density difference along the plume axis.

Council, Outstanding Young Researcher Award, Grant VR 2014-5001 (L. B.). Support to C. C. was given by the NSF Project OCE-1434041. Computer time was provided by the Swedish National Infrastructure for Computing (SNIC). Visualization and graphic analysis were performed with VisIt (Childs et al. 2012) and Gnuplot.

\section{APPENDIX A}

\section{Validation: A Lazy Plume in a Homogeneous Fluid}

To validate our implementation we have performed a simulation of a lazy plume in a homogeneous fluid of density equal to the water density at the seabed. The parameters in the simulation are as follows: the source diameter $D_{h}=D_{0}=20 \mathrm{~m}$, the initial velocity $W_{h 0}=1.1 \mathrm{~m} \mathrm{~s}^{-1}$, density of the plume $\rho_{h 0}=1.002 \mathrm{~g} \mathrm{~cm}^{-3}$, and density of the ambient fluid $\rho_{h a}=1.0307 \mathrm{~g} \mathrm{~cm}^{-3}$. We performed the simulation in a rectangular domain with size $30 D_{h} \times$ $30 D_{h} \times 45 D_{h}$ and compared the results with theory, experiments, and previous direct numerical simulation (DNS) and LES (Pham et al. 2007; Plourde et al. 2008; Pham et al. 2006).

The lazy plume considered here has nonzero volume and momentum and buoyancy fluxes. Following Kaye
(2008), one may introduce several length scales characterizing the plume depending on these fluxes, namely, a source-based plume diameter, Morton length scale (or the jet length scale), as well as the acceleration length scale. The jet length scale corresponds to the distance from the source where the plume reaches the balance between buoyancy and momentum fluxes and exhibits the behavior typical of pure plumes. We estimate the jet length scale for the lazy plume considered here as $L_{M} \sim D_{h} \mathrm{Fr}_{h} \sim D_{h}$. Figure A1 displays the mean velocity and density variation along the plume axis. They follow the theoretical predictions $U_{c} \sim z^{-1 / 3}$ and $\rho_{c} \sim z^{-5 / 3}$ for a pure plume for $z>15$. According to experiments (Shabbir and George 1994), the behavior typical of a pure plume is reached after $\sim 5 L_{M}$; thus, we expect it for $z>5$. The curves presented here demonstrate this behavior after $\sim 15 L_{M}$, that is, it takes somewhat longer to establish the plume behavior in LES. This is however typical for all the LES models used to simulate buoyant plumes (see, e.g., Pham et al. 2007).

Figure 5 shows the variation of the plume radius with the vertical coordinate. The plume radius is defined as the length scale where the mean vertical velocity falls $e$ times as compared to the velocity on the plume axis. According to the theoretical predictions based on conservation laws, the dependence of the radius on the 

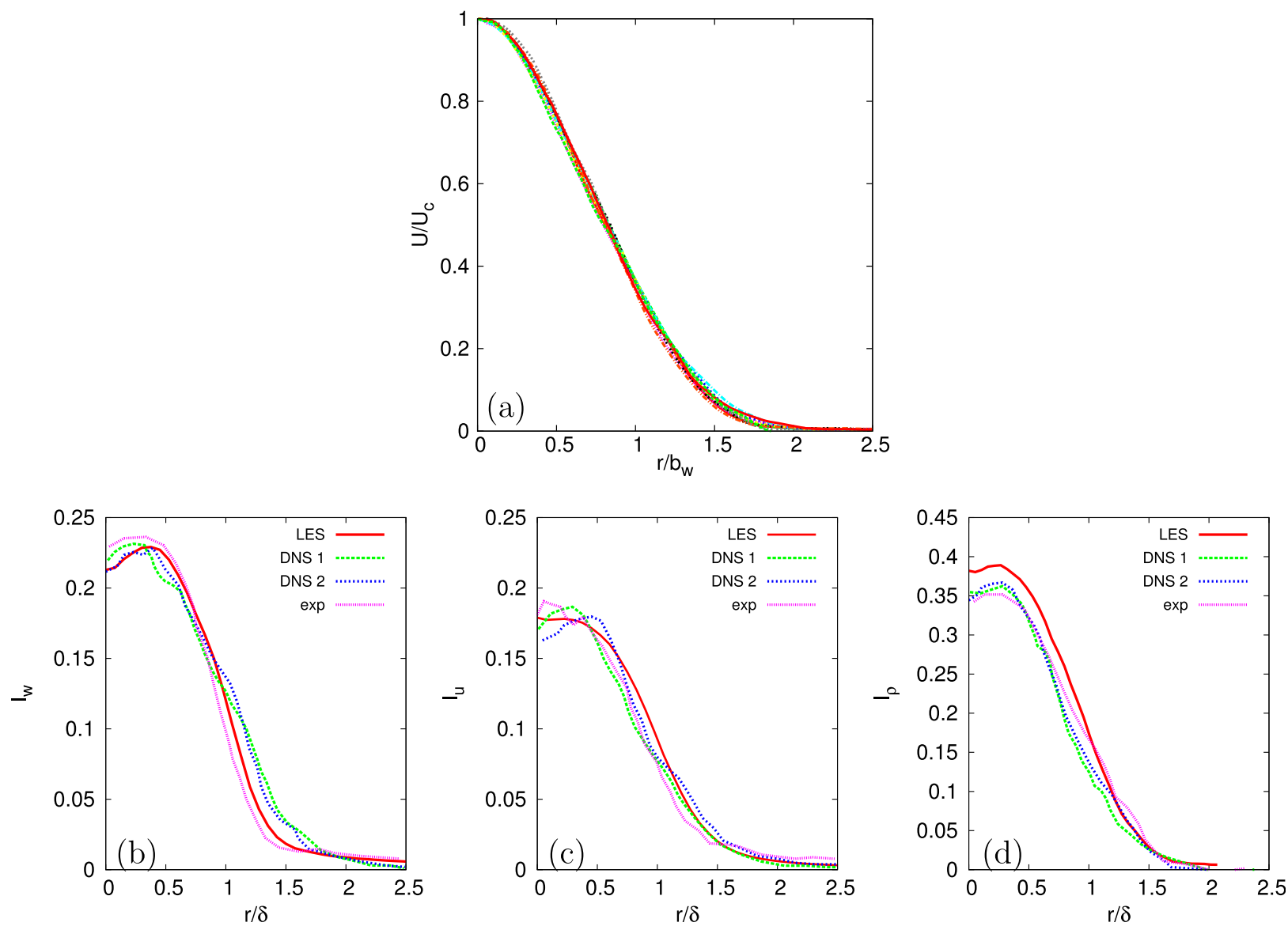

FIG. A2. (a) Nondimensional radial profiles of time-averaged vertical velocity at $10 z$ cross sections: $4.4<z<34.8$. (bottom) Nondimensional turbulent intensity of (b) axial and (c) radial velocity component, and (d) density profiles averaged over several $z$ cross sections: $10.4<z<34.8$. LES data are compared to the DNS and experimental results by Plourde et al. (2008) and Pham et al. (2006).

vertical coordinate should be linear, $b_{w}=6 / 5 \alpha_{p g} z$, with the experimentally established value of the entrainment coefficient $\alpha_{p g}$ in the range between 0.07 and 0.11 . The values based on our simulations demonstrate a welldefined linear dependence with $\alpha_{p g}=0.081$, that is, within this range.

Figure A2 shows the mean vertical velocity profile as well as the rms of velocity and density fluctuations in self-similar coordinates: $I_{u}=\sqrt{\tilde{u}_{r}^{\prime 2} / U_{c}}, I_{w}=\sqrt{\tilde{u}_{z}^{2}} / U_{c}$, and $I_{\rho}=\sqrt{\tilde{\rho}^{2}} /\left(\rho_{a}-\rho_{c}\right)$. The length scale in the lower panel of Fig. A2 is defined following Plourde et al. (2008): $\delta^{2}(z)=2\left(\int_{0}^{\infty} U r d r\right)^{2} / \int_{0}^{\infty} U^{2} r d r$. The results of our LES agree well with the results of DNS and experiments (Plourde et al. 2008; Pham et al. 2006), thus validating the dynamic Smagorinsky model in the code Nek5000 for the simulations of buoyant plumes. The level of density fluctuations on the axis is a bit higher here than in the data from DNS and the experimental results. The DNS profiles are taken by Pham et al. (2007) at the two levels where the intensity of the fluctuations on the axis is close to the lowest levels. However, their Fig. 8 shows that the measured intensity reaches 0.38 at least on half of their experimental points, which is in closer agreement with our results.

\section{APPENDIX B}

\section{Nondimensional Parameters of Simulations}

To make the comparison between the different cases easier, we make the equations nondimensional with the same density difference (the difference between the bottom and the surface in winter $\Delta \rho=1.03070-1.02697=$ $0.00373 \mathrm{~g} \mathrm{~cm}^{-3}$ ) and the same length scale $D_{0}=20 \mathrm{~m}$ (the larger diameter of the source), while the velocity scale corresponds to the inflow velocity of each particular case. The reference density value is $\rho_{\text {ref }}=1.02697 \mathrm{~g} \mathrm{~cm}^{-3}$ for all the cases, which is the density of the fluid at the surface in winter. The temperature of the source fluid is taken equal to the freezing temperature of freshwater at the depth $600 \mathrm{~m}$ 
$\left(T_{0}=-4.5^{\circ} \mathrm{C}\right)$. For these parameters $\rho_{0}=1.00251 \mathrm{~g} \mathrm{~cm}^{-3}$. Thus, in winter, the nondimensional density on the surface is 0 , on the bottom is 1 , and the density of the plume is -6.5 . In summer, instead, we have the fluid density on the surface $1.0205 \mathrm{~g} \mathrm{~cm}^{-3}$, corresponding to $\approx-1.73$ in nondimensional variables. The analytical functions we used for the approximation of the density profiles are as follows:

in winter,

$$
\begin{aligned}
\rho_{s}^{\prime}= & 0.0257 \times(H-z)+0.5 \times 0.23 \\
& \times\{1-\tanh [0.46(z-21.6)]\},
\end{aligned}
$$

in summer,

$$
\begin{aligned}
\rho_{s}^{\prime}= & 0.0257 \times(H-z)-69.36193 \\
& +0.5 \times 1.71 \times\{1-\tanh [2.58(z-29.773)]\} \\
& +0.5 \times 67.882 \times\{1-\tanh [0.146(z-45.25)]\},
\end{aligned}
$$

where $z=0$ corresponds to the bottom and $z=H$ corresponds to the surface.

The velocity profile is given by a slightly smoothed step function (the width of the step is 0.05 ). The nondimensional diameter corresponding to a $20-\mathrm{m}$ source is 1 , while the nondimensional diameter for a $10-\mathrm{m}$ source is 0.5 .

\section{REFERENCES}

Ansong, J. K., and B. R. Sutherland, 2010: Internal gravity waves generated by convective plumes. J. Fluid Mech., 648, 405-434, doi:10.1017/S0022112009993193.

Bamber, J., M. van den Broeke, J. Ettema, J. Lenaerts, and E. Rignot, 2012: Recent large increases in freshwater fluxes from Greenland into the North Atlantic. Geophys. Res. Lett., 39, L19501, doi:10.1029/2012GL052552.

Burridge, H. C., G. R. Partridge, and P. F. Linden, 2016: The fluxes and behaviour of plumes inferred from measurements of coherent structures within images of the bulk flow. Atmos.-Ocean, 54, 403-417, doi:10.1080/07055900.2016.1175337.

Cardoso, S. S. S., and A. W. Woods, 1993: Mixing by a turbulent plume in a confined stratified region. J. Fluid Mech., 250, 277-305, doi:10.1017/S0022112093001466.

Carroll, D., and Coauthors, 2016: The impact of glacier geometry on meltwater plume structure and submarine melt in Greenland fjords. Geophys. Res. Lett., 43, 9739-9748, doi:10.1002/2016GL070170.

Cenedese, C., and V. M. Gatto, 2016a: Impact of a localized source of subglacial discharge on the heat flux and submarine melting of a tidewater glacier: A laboratory study. J. Phys. Oceanogr., 46, 3155-3163, doi:10.1175/JPO-D-16-0123.1.

$\longrightarrow$, and $-2016 \mathrm{~b}$ : Impact of two plumes, interaction on submarine melting of tidewater glaciers: A laboratory study. J. Phys. Oceanogr., 46, 361-367, doi:10.1175/JPO-D-15-0171.1.

Childs, H., and Coauthors, 2012: VisIt: An end-user tool for visualizing and analyzing very large data. High Performance Visualization: Enabling Extreme-Scale Scientific Insight, CRC Press, 357-372.

Cowton, T., D. Slater, A. Sole, D. Goldberg, and P. Nienow, 2015: Modeling the impact of glacial runoff on fjord circulation and submarine melt rate using a new subgrid-scale parameterization for glacial plumes. J. Geophys. Res. Oceans, 120, 796-812, doi:10.1002/2014JC010324.

Dimotakis, P., 2005: Turbulent mixing. Annu. Rev. Fluid Mech., 37, 329-356, doi:10.1146/annurev.fluid.36.050802.122015.

Ezhova, E., C. Cenedese, and L. Brandt, 2016: Interaction of vertical turbulent jets with a thermocline. J. Phys. Oceanogr., 46, 3415-3437, doi:10.1175/JPO-D-16-0035.1.

Ezzamel, A., P. Salizzoni, and G. Hunt, 2015: Dynamical variability of axisymmetric buoyant plumes. J. Fluid Mech., 765, 576-611, doi:10.1017/jfm.2014.694.

Fabregat, A., A. C. Poje, T. M. Özgökmen, and W. K. Dewar, 2016: Dynamics of multiphase turbulent plumes with hybrid buoyancy sources in stratified environments. Phys. Fluids, 28, 095109, doi:10.1063/1.4963313.

Fischer, H. B., E. List, R. Koh, J. Imberger, and N. Brooks, 1979: Mixing in Inland and Coastal Waters. Academic Press, 483 pp.

Fischer, P. F., J. W. Lottes, and S. G. Kerkemeier, 2008: Nek5000: A fast and scalable open-source spectral element solver for CFD. Argonne National Laboratory Mathematics and Computer Science Division, http://nek5000.mcs.anl.gov.

Gardner, A. S., and Coauthors, 2013: A reconciled estimate of glacier contributions to sea level rise: 2003 to 2009. Science, 340, 852-857, doi:10.1126/science.1234532.

Germano, M., U. Piomelli, P. Moin, and W. H. Cabot, 1991: A dynamic subgrid-scale eddy viscosity model. Phys. Fluids, 3A, 1760-1765, doi:10.1063/1.857955.

Gill, A. E., 1982: Atmosphere-Ocean Dynamics. Academic Press, 662 pp.

Holland, D. M., and A. Jenkins, 1999: Modeling thermodynamic ice-ocean interactions at the base of an ice shelf. J. Phys. Oceanogr., 29, 1787-1800, doi:10.1175/1520-0485(1999)029<1787: MTIOIA $>2.0 . C O ; 2$.

Hunt, G. R., and N. B. Kaye, 2001: Virtual origin correction for lazy turbulent plumes. J. Fluid Mech., 435, 377-396, doi:10.1017/ S0022112001003871.

_, and —, 2005: Lazy plumes. J. Fluid Mech., 533, 329-338, doi:10.1017/S002211200500457X.

Jenkins, A., 2011: Convection-driven melting near the grounding lines of ice shelves and tidewater glaciers. J. Phys. Oceanogr., 41, 2279-2294, doi:10.1175/JPO-D-11-03.1.

Kaye, N. B., 2008: Turbulent plumes in stratified environments: A review of recent work. Atmos.-Ocean, 46, 443-441.

Kimura, S., P. Holland, A. Jenkins, and M. Piggott, 2014: The effect of meltwater plumes on the melting of a vertical glacier face.J. Phys. Oceanogr., 44, 3099-3117, doi:10.1175/JPO-D-13-0219.1.

Mankoff, K. D., F. Straneo, C. Cenedese, S. B. Das, C. D. Richards, and H. Singh, 2016: Structure and dynamics of a subglacial discharge plume in a Greenland fjord. J. Geophys. Res. Oceans, 121, 8670-8688, doi:10.1002/2016JC011764.

McConnochie, C. D., and R. Kerr, 2017: Enhanced ablation of a vertical ice wall due to an external freshwater plume. J. Fluid Mech., 810, 429-447, doi:10.1017/jfm.2016.761.

Morton, B. R., G. Taylor, and J. S. Turner, 1956: Turbulent gravitational convection from maintained and instantaneous sources. Proc. Roy. Soc. London, A234, 1-25, doi:10.1098/ rspa.1956.0011.

Özgökmen, T. M., and P. F. Fischer, 2008: On the role of bottom roughness in overflows. Ocean Modell., 20, 336-361, doi:10.1016/j.ocemod.2007.10.004.

, T. Iliescu, and P. F. Fischer, 2009: Large eddy simulation of stratified mixing in a three-dimensional lock-exchange system. Ocean Modell., 26, 134-155, doi:10.1016/ j.ocemod.2008.09.006. 
Pham, M. V., F. Plourde, and K. S. Doan, 2006: Effect of swirl on pure turbulent thermal plume development. Int. J. Heat Fluid Flow, 27, 502-513, doi:10.1016/j.ijheatfluidflow.2005.12.001.

_ — - and — 2007: Direct and large-eddy simulations of a pure thermal plume. Phys. Fluids, 19, 125103, doi:10.1063/ 1.2813043.

Plourde, F., M. V. Pham, K. S. Doan, and S. Balachandar, 2008: Direct numerical simulations of a rapidly expanding thermal plume: Structure and entrainment interaction. J. Fluid Mech., 604, 99-123, doi:10.1017/S0022112008001006.

Sciascia, R., F. Straneo, C. Cenedese, and P. Heimbach, 2013: Seasonal variability of submarine melt rate and circulation in an east Greenland fjord. J. Geophys. Res. Oceans, 118, 2492-2506, doi:10.1002/jgrc.20142.

Shabbir, A., and W. K. George, 1994: Experiments on a round turbulent buoyant plume. J. Fluid Mech., 275, 1-32, doi:10.1017/ S0022112094002260.

Shepherd, A., and Coauthors, 2012: A reconciled estimate of ice-sheet mass balance. Science, 338, 1183-1189, doi:10.1126/science.1228102.

Shrinivas, A. B., and G. R. Hunt, 2014: Unconfined turbulent entrainment across density interfaces. J. Fluid Mech., 757, 573-598, doi:10.1017/jfm.2014.474.

Slater, D. A., P. W. Nienow, T. R. Cowton, D. N. Goldberg, and A. J. Sole, 2015: Effect of near-terminus subglacial hydrology on tidewater glacier submarine melt rates. Geophys. Res. Lett., 42, 2861-2868, doi:10.1002/2014GL062494.

Smagorinsky, J., 1963: General circulation experiments with the primitive equations. Mon. Wea. Rev., 91, 99-164, doi:10.1175/ 1520-0493(1963)091<0099:GCEWTP>2.3.CO;2.
Straneo, F., and C. Cenedese, 2015: Dynamics of Greenland's glacial fjords and their role in climate. Annu. Rev. Mar. Sci., 7, 89-112, doi:10.1146/ annurev-marine-010213-135133.

- R. Curry, D. Sutherland, G. Hamilton, C. Cenedese, K. Vage, and L. Stearns, 2011: Impact of fjord dynamics and glacial runoff on the circulation near Helheim Glacier. Nat. Geosci., 4, 322-327, doi:10.1038/ngeo1109.

— , and Coauthors, 2012: Characteristics of ocean waters reaching Greenland's glaciers. Ann. Glaciol., 53, 202-210, doi:10.3189/2012AoG60A059.

Troitskaya, Y. I., D. A. Sergeev, E. V. Ezhova, I. A. Soustova, and V. I. Kazakov, 2008: Self-induced internal waves excited by buoyant plumes in a stratified tank. Dokl. Earth Sci., 419 , 506-510, doi:10.1134/S1028334X08030343.

van Reeuwijk, M., P. Salizzoni, G. R. Hunt, and J. Craske, 2016: Turbulent transport and entrainment in jets and plumes: A DNS study. Phys. Rev. Fluids, 1, 074301, doi:10.1103/ PhysRevFluids.1.074301.

Xu, Y., E. Rignot, D. Menemenlis, and M. Koppes, 2012: Numerical experiments on subaqueous melting of Greenland tidewater glaciers in response to ocean warming and enhanced subglacial discharge. Ann. Glaciol., 53, 229-234, doi:10.3189/ 2012AoG60A139.

— — - I. Fenty, D. Menemenlis, and M. M. Flexas, 2013: Subaqueous melting of Store Glacier, west Greenland from three-dimensional, high-resolution numerical modeling and ocean observations. Geophys. Res. Lett., 40, 4648-4653, doi:10.1002/grl.50825. 